\title{
EFFICIENCY DECOMPOSITION IN A THREE-STAGE NETWORK STRUCTURE: COOPERATIVE DEA, NASH BARGAINING GAME MODELS AND CONIC RELAXATIONS
}

\author{
Narges Torabi Golsefid ${ }^{1}$ And Maziar SAlahi ${ }^{2, *}$
}

\begin{abstract}
In this paper, for evaluating the efficiency in a three-stage DEA structure we use the additive and the multiplicative cooperative models that comply with the cooperation paradigm in the organizations, where for improving efficiency of system, stages cooperate together. Since the overall efficiency from the cooperative models may not be unique and consequently the stages' efficiencies, then we combine them with the Nash bargaining game approach that besides maximizing efficiency scores for stages and the whole system, provides a unique and fair efficiency decomposition. Second order programming relaxation of the proposed nonlinear models are given in contrast to the parametric linear models in the literature. Finally, the effectiveness of the proposed models are illustrated with two numerical examples.
\end{abstract}

Mathematics Subject Classification. 90B30, 91A40, 90C25.

Received November 1, 2019. Accepted November 21, 2021.

\section{INTRODUCTION}

Since performance improvement is an important target of managers at each organization, it is crucial to use appropriate criteria to make the best decision. Data envelopment analysis (DEA) is one of the most popular performance measurement methods introduced by Charnes et al. [8]. It is a non-parametric approach to determine the relative efficiency of peer decision-making units (DMUs) without the need to the production function. Original DEA models consider only the inputs consumed by and the outputs produced from the system. In other words, they treat the system as a black box, so its internal operations are neglected in measuring efficiency. Intermediate products connect stages together and play a key role in the efficiency assessment in the network structures. Fare et al. $[17,18]$ were among the first to study the system efficiency in such structures. To identify the relationship between the overall efficiency and the stages efficiencies, several models are proposed in the DEA literature $[11,13,25,27]$. Decomposition of the production process in the network structure helps to identify the sources of inefficiency in the system. One popular approach for the efficiency decomposition in the network structures is multiplicative efficiency decomposition (MED) approach introduced by Kao et al. [25] that defines

Keywords. Cooperative models, data envelopment analysis, three-stage models, Nash bargaining game, conic relaxation.

1 Department of Applied Mathematics, Faculty of Mathematical Sciences, University of Guilan, Rasht, Iran.

2 Department of Applied Mathematics, Faculty of Mathematical Sciences, and Center of Excellence for Mathematical Modeling, Optimization and Combinatorial Computing (MMOCC), University of Guilan, Rasht, Iran.

*Corresponding author: salahim@guilan.ac.ir; salahi.maziar@gmail.com

(C) The authors. Published by EDP Sciences, ROADEF, SMAI 2021 
the overall efficiency in the two-stage process as the product of stages' efficiencies. The other one is the additive efficiency decomposition (AED) introduced by Chen et al. [10]. They pointed out some weaknesses of MED and as an alternative defined the overall efficiency as the weighted sum of stages' efficiencies. In both approaches, the stages' efficiencies and the overall efficiency are estimated, separately. Since MED may not lead to a unique decomposition, Despotis et al. [14] used a multi-objective programming approach in a multi-stage processes to resolve this issue. However, in their proposed approach, there is no relation between the overall efficiency and the stages' efficiencies. In addition, in the overall efficiency determination intermediate products play no role.

Extensions to multi-stage structures are given by Castelli et al. [5]. In two different studies, Kao [22, 23] discussed the efficiency measurement and decomposition of general multi-stage structures. However, both studies limited to the assumption of constant returns to scale (CRS). To evaluate the efficiency in a three-stage structure and to obtain a unique solution, Chao [6] used a two-phase algorithm and a fuzzy analytical hierarchical process. Kao [24] presented a classification of slacks-based efficiency measures in a three-stage network DEA. He showed that the cooperative type is appropriate for both external and internal evaluations of system. Zhang et al. [43] studied multi-stage network DEA with semidefinite programming approach. They also extended multistage processes with feedbacks based on additive efficiency decomposition. In another study, using efficiency evaluations over consecutive periods of a three-stage system, Zhou et al. [44] identified more detailed information of stages inefficiencies reasons. To evaluate the performance and to identify the projection points of bank branches in a three-stage structure, Mahmoudabadi et al. [32] used a slack-based measure (SBM) DEA model. Due to the existence of slacks in the SBM model, it determines the furthest efficient projections on the frontier for the inefficient DMUs which leads to determination of unrealistic and unreachable targets for the inputs and outputs such that the assessed inefficient DMU probably cannot achieve its targets [2].

To deal with network DEA models, there are two different approaches from the perspective of game theory: cooperative [15] and non-cooperative [35], where a stage makes a choice by considering the choice of other stages. In the cooperative models, the overall efficiency of system is estimated first and then the stages efficiencies are obtained. In other words, the system is evaluated using the set of common weights. Also, in the cooperative models, unlike the non-cooperative models, the stages have no priority, that means their importance degrees are equal and the focus is on maximizing the overall efficiency of system and the efficiency scores of stages. Indeed to improve the overall efficiency of the whole system, all stages cooperate together. To show the conflict between the stages, Liang et al. [29] examined the relation among the non-cooperative, cooperative and standard DEA approaches. To achieve a unique solution in simple network structure without the exogenous inputs and outputs, they used the leader-follower approach, where the priority is with the leader stage. Also, they showed that the overall efficiency is the product of the stages' efficiencies in the two-satge. Using the non-cooperative approach, Tavana et al. [36] proposed an efficienct two-stage fuzzy DEA model to calculate the overall efficiency and the stages' efficiencies. To provide an approach for analyzing the reuse of undesirable intermediate outputs in a two-stage production process with shared resources, Wu et al. [40] proposed additive efficiency measures and non-cooperative effciency measures. In another study, Tavana et al. [37] presented a two-stage DEA model to evaluate the performance that can be easily implemented for comprehensive analysis of multi-level supply chains. To generate a pareto solution and identify the leader stage, Li et al. [28] extended the work by Despoits et al. [14]. They showed that the optimal solution for the extended model is also a non-cooperative solution.

All the abovementioned models may result in non-unique efficiency decomposition, thus in order to obtain a fair and unique efficiency and no precondition in the multi-stage structures, the Nash bargaining game approach is used [33,34]. For measuring performance of a basic two-stage network, Du et al. [16] proposed a nonlinear Nash bargaining game model. They first constructed the least ideal DMU and used its DEA efficiency score as the breakdown point. Then, they showed that their nonlinear model can be converted into a parametric linear programming problem where the optimal solution is obtained using a heuristic search. Since multiple optimal weights may lead to some flexibility in decomposing the overall efficiency into the stages' efficiencies in the cooperative model, Zhou et al. [45] developed a Nash bargaining game model for a basic two-stage network DEA to obtain a fair efficiency decomposition, while keeping the overall efficiency unchanged. They used the minimum achievable efficiencies of the two stages as the breakdown points. However, none of the 
two latter studies considered the exogenous inputs and outputs in the network structure. Also, models of both latter studies can only deal with the assumption of CRS. To make an equilibrium between desirable outputs and undesirable outputs in a two-stage sustainable manufacturing process, Wu et al. [41] proposed a Nash bargaining game model. Recently, using the cooperative approach in a three-stage network with external inputs and outputs, Amirkhan et al. [1] decomposed the overall efficiency into the stages' efficiencies. They developed a three-stage DEA model based on the Nash bargaining game that provided a unique and fair decomposition of the overall efficiency among the stages. To solve the proposed nonlinear bargaining model, they utilized a heuristic search approach. However, to calculate the overall efficiency in the three-stage structure with external inputs and outputs, their proposed method has neglected the existence of intermediate products. Using the concept borrowed from Nash bargaining game, Mahmoudi et al. [31] also handled insufficient number of DMUs and inputs issue in the network structure.

Utilizing the exogenous inputs and outputs in the network structure, such as the outputs that are sent directly to the outside from the first stage and the additional inputs to intermediate stages, make the multi-stage DEA models highly nonlinear. Applying Charnes-Cooper transformations to such models usually lead to parametric linear programs for which heuristic methods often are used [27]. To surmount this issue, Chen et al. [9] developed a second-order cone program (SOCP) approach to solve nonlinear two-stage DEA models. Their approach only ensures a feasible solution instead of the global optimal solution. Recently in another study, Chen et al. [12] pointed out weakness of Chen et al. [9]'s approach and introduced a conic relaxation model that generates feasible approximations and tighter upper bounds on the overall efficiency. In this paper, we consider a more general form of the three-stage structure studied by Amirkhan et al. [1] and Despotis et al. [14] based on the AED and MED in the cooperative game context. Our first aim is to maximize the stages' efficiencies and the overall efficiency of such structures. First, we maximize the overall efficiency in both AED and MED models, then the stages' efficiencies are derived from the weights that are obtained by AED and MED models. The second aim of this paper is to achieve a fair and unique efficiency decomposition of the overall efficiency into the stages' efficiencies. To this end, we utilize the Nash bargaining game approach. Since the proposed models cannot be linearized using Charnes-Cooper transformation [7], we present their SOCP relaxations. To sum up, compared with the previous studies in the multi-stage structure, the proposed approaches in this paper has four merits. First, we use network structure under variable returns to scale (VRS). Second, to maximize all stages' efficiencies, we study both AED and MED under cooperative relationship between the three stages of system. Each stage in the cooperative approach tries to achieve weights to maximize its efficiency, but cannot effect the weights of the other stages. In other words, each stage's weights are independent of the other stages' weights. Third, there are structures where the stages' weights may depend on the weights of the other stages. Thus, to make an equilibrium between stages' efficiencies, we utilize Nash bargaining game approach. Fourth, to solve the three nonlinear models, we use SOCP relaxations.

We begin Section 2 with the conventional BCC model on the three-stage structure and continue with the introduction of models based on cooperative game approach and their SOCP formulations. In Section 3, we propose the Nash bargaining game model to improve the stages' efficiencies, and present its SOCP formulation. In Section 4, we generalize the proposed bargaining model to the multi-stage structure. In Section 5, two numerical examples are used to compare the proposed models. Finally, the conclusions are given in the last section. The notations throughout the paper are summarized in Table 1.

\section{Cooperative efficiency evaluation in a three-Stage System}

Consider the three-stage serial structure as depicted in Figure 1. The conventional BCC model for measuring the whole system performance under VRS can be expressed as follows [3]:

$$
E_{o}^{\mathrm{BCC}}=\max \frac{u^{1} \cdot y_{o}^{1}+u^{2} \cdot y_{o}^{2}+u^{3} \cdot y_{o}^{3}+u_{0}}{v^{1} \cdot x_{o}^{1}+v^{2} \cdot x_{o}^{2}+v^{3} \cdot x_{o}^{3}}
$$

s.t. 
TABLE 1. Notations.

\begin{tabular}{ll}
\hline \hline Notation & Description \\
\hline$n$ & Number of DMUs \\
$m_{k}$ & Number of exogenous inputs of stage $k$ \\
$s_{k}$ & Number of exogenous outputs of stage $k$ \\
$l_{k}$ & Number of intermediate products of stage $k$ \\
$x_{i j}^{k}$ & $i$ th input of $\mathrm{DMU}_{j}$ of stage $k$ \\
$y_{r j}^{k}$ & $r$ th output of $\mathrm{DMU}_{j}$ of stage $k$ \\
$z_{d j}^{k}$ & $d$ th intermediate product of DMU of stage $k$ \\
$v_{i}^{k}$ & Weight assigned to the $i$ th input of stage $k$ \\
$u_{r}^{k}$ & Weight assigned to the $r$ th output of stage $k$ \\
$w_{d}^{k}$ & Weight assigned to the $d$ th intermediate product of stage $k$ \\
$a \cdot b$ & Inner product of vectors $a$ and $b$ \\
$a$ & Componentwise division of vectors $a$ and $b i . e ., \frac{a_{i}}{b_{i}}$ \\
$X$ & The inputs matrix of all DMUs \\
$Z$ & The intermediate products matrix of all DMUs \\
$Y$ & The outputs matrix of all DMUs \\
\hline
\end{tabular}

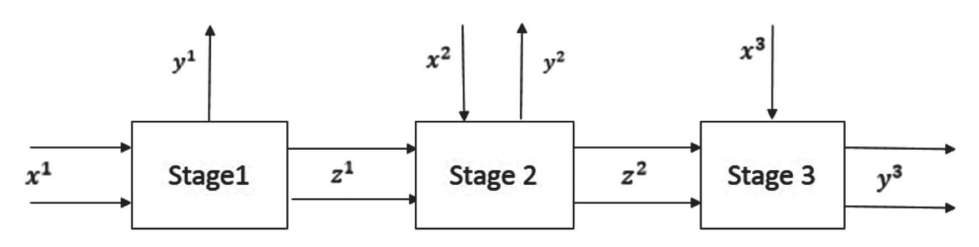

Figure 1. Three-stage system.

$$
\begin{aligned}
& \frac{Y^{1} u^{1}+Y^{2} u^{2}+Y^{3} u^{3}+u_{0}}{X^{1} v^{1}+X^{2} v^{2}+X^{3} v^{3}} \leq 1, \\
& v^{1}, v^{2}, v^{3}, u^{1}, u^{2}, u^{3} \geq 0 ; u_{0} \text { is free. }
\end{aligned}
$$

If we set $u_{0}=0$, then model (2.1) converts to the CCR model [8]. Indeed, this variable characterizes the intercept and relaxes the condition that the production function must pass from the orgin [26]. Obviously, intermediate products produced and consumed within the system are not considered in model (2.1), and system's performance is measured from the viewpoint of outside. Since system's performance depends on the intermediate products that are not observable from outside and their weak performance may lead to overall inefficiency of system, thus we measure efficiency from the viewpoint of inside. To evaluate the overall efficiency of DMU under evaluation $\left(\mathrm{DMU}_{o}\right)$ according to $\mathrm{AED}$, we consider the weighted sum of stages' efficiencies as follows:

$$
\begin{aligned}
E_{o}^{\mathrm{AC}}=\max & \alpha \frac{w^{1} \cdot z_{o}^{1}+u^{1} \cdot y_{o}^{1}+u_{0}}{v^{1} \cdot x_{o}^{1}}+\beta \frac{w^{2} \cdot z_{o}^{2}+u^{2} \cdot y_{o}^{2}+w_{0}}{w^{1} \cdot z_{o}^{1}+v^{2} \cdot x_{o}^{2}}+\gamma \frac{u^{3} \cdot y_{o}^{3}+v_{0}}{w^{2} \cdot z_{o}^{2}+v^{3} \cdot x_{o}^{3}} \\
\text { s.t. } & \frac{Z^{1} w^{1}+Y^{1} u^{1}+u_{0}}{X^{1} v^{1}} \leq 1,
\end{aligned}
$$




$$
\begin{aligned}
& \frac{Z^{2} w^{2}+Y^{2} u^{2}+w_{0}}{Z^{1} w^{1}+X^{2} v^{2}} \leq 1 \\
& \frac{Y^{3} u^{3}+v_{0}}{Z^{2} w^{2}+X^{3} v^{3}} \leq 1 \\
& v^{1}, v^{2}, v^{3}, u^{1}, u^{2}, u^{3}, w^{1}, w^{2} \geq 0 ; u_{0}, w_{0}, v_{0} \text { are free }
\end{aligned}
$$

where $0 \leq \alpha, \beta, \gamma \leq 1$ are the stages' weights. The overall efficiency score for $\mathrm{DMU}_{o}$ using the AED network DEA model (2.2) relies on the predetermined weights which is either provided by the decision-makers or determined by the model. Since cooperative approach considers same priorities for the stages, thus we consider $\alpha=\beta=\gamma=\frac{1}{3}$ in model (2.2). Further, since model (2.2) is nonlinear, we present its conic relaxation formulation. The following lemma play key roles in the conic relaxation formulations.

Lemma $2.1([4]) \cdot w^{2} \leqslant u v, v, u \geq 0 \Leftrightarrow\left\|\left[\begin{array}{c}2 w \\ u-v\end{array}\right]\right\| \leqslant u+v$.

In the sequel, inspired by Chen [12], a conic relaxation of model (2.2) is given. To do so, suppose that $\left(v^{1 *}, v^{2 *}, v^{3 *}, u^{1 *}, u^{2 *}, u^{3 *}, w^{1 *}, w^{2 *}, u_{0}^{*}, w_{0}^{*}, v_{0}^{*}\right)$ is an optimal solution of model (2.2). Let $\eta=$ $\max \left\{v^{1 *} \cdot x_{o}^{1}, w^{1 *} \cdot z_{o}^{1}+v^{2 *} \cdot x_{o}^{2}, w^{2 *} \cdot z_{o}^{2}+v^{3 *} \cdot x_{o}^{3}\right\}$, then $\left(\frac{v^{1 *}}{\eta}, \frac{v^{2 *}}{\eta}, \frac{v^{3 *}}{\eta}, \frac{u^{1 *}}{\eta}, \frac{u^{2 *}}{\eta}, \frac{u^{3 *}}{\eta}, \frac{w^{1 *}}{\eta}, \frac{w^{2 *}}{\eta}, \frac{u_{0}^{*}}{\eta}, \frac{w_{0}^{*}}{\eta}, \frac{v_{0}^{*}}{\eta}\right)$ is still an optimal solution and the overall efficiency remains unchanged. On the other hand, $\frac{v^{1 *}}{\eta} \cdot x_{o}^{1}, \frac{w^{1 *}}{\eta} \cdot z_{o}^{1}+$ $\frac{v^{2 *}}{\eta} \cdot x_{o}^{2}$ and $\frac{w^{2 *}}{\eta} \cdot z_{o}^{2}+\frac{v^{3 *}}{\eta} \cdot x_{o}^{3}$ are now between zero and one. Thus, we add the following constraints

$$
\begin{aligned}
& 0 \leq w^{1} \cdot z_{o}^{1}+u^{1} \cdot y_{o}^{1}+u_{0} \leq 1, \\
& 0 \leq v^{1} \cdot x_{o}^{1} \leq 1 \\
& 0 \leq w^{2} \cdot z_{o}^{2}+u^{2} \cdot y_{o}^{2}+w_{0} \leq 1, \\
& 0 \leq w^{1} \cdot z_{o}^{1}+v^{2} \cdot x_{o}^{2} \leq 1 \\
& 0 \leq u^{3} \cdot y_{o}^{3}+v_{0} \leq 1 \\
& 0 \leq w^{2} \cdot z_{o}^{2}+v^{3} \cdot x_{o}^{3} \leq 1
\end{aligned}
$$

to model (2.2) without losing its optimal solution. Thus in the sequel, we consider the following model:

$$
\begin{aligned}
E_{o}^{\mathrm{AC}}=\max & \alpha \frac{w^{1} \cdot z_{o}^{1}+u^{1} \cdot y_{o}^{1}+u_{0}}{v^{1} \cdot x_{o}^{1}}+\beta \frac{w^{2} \cdot z_{o}^{2}+u^{2} \cdot y_{o}^{2}+w_{0}}{w^{1} \cdot z_{o}^{1}+v^{2} \cdot x_{o}^{2}}+\gamma \frac{u^{3} \cdot y_{o}^{3}+v_{0}}{w^{2} \cdot z_{o}^{2}+v^{3} \cdot x_{o}^{3}} \\
\text { s.t. } & \\
& \frac{Z^{1} w^{1}+Y^{1} u^{1}+u_{0}}{X^{1} v^{1}} \leq 1, \\
& \frac{Z^{2} w^{2}+Y^{2} u^{2}+w_{0}}{Z^{1} w^{1}+X^{2} v^{2}} \leq 1, \\
& \frac{Y^{3} u^{3}+v_{0}}{Z^{2} w^{2}+X^{3} v^{3}} \leq 1, \\
& 0 \leq w^{1} \cdot z_{o}^{1}+u^{1} \cdot y_{o}^{1}+u_{0} \leq 1, \\
& 0 \leq v^{1} \cdot x_{o}^{1} \leq 1, \\
& 0 \leq w^{2} \cdot z_{o}^{2}+u^{2} \cdot y_{o}^{2}+w_{0} \leq 1, \\
& 0 \leq w^{1} \cdot z_{o}^{1}+v^{2} \cdot x_{o}^{2} \leq 1, \\
& 0 \leq u^{3} \cdot y_{o}^{3}+v_{0} \leq 1,
\end{aligned}
$$




$$
\begin{aligned}
& 0 \leq w^{2} \cdot z_{o}^{2}+v^{3} \cdot x_{o}^{3} \leq 1 \\
& v^{1}, v^{2}, v^{3}, u^{1}, u^{2}, u^{3}, w^{1}, w^{2} \geq 0 ; u_{0}, w_{0}, v_{0} \text { are free. }
\end{aligned}
$$

Theorem 2.2. Conic relaxation of additive model (2.4) is as follows:

$$
\begin{aligned}
& \min \alpha \theta_{1}+\beta \theta_{2}+\gamma \theta_{3} \\
& \text { s.t. } \\
& \begin{array}{ll}
u_{i}^{o} s_{i}+t_{i} U_{i}^{o}-u_{i}^{o} U_{i}^{o} \leq \theta_{i}, & i=1,2,3, \\
t_{i} L_{i}^{o}+l_{i}^{o} s_{i}-l_{i}^{o} L_{i}^{o} \leq \theta_{i}, & i=1,2,3,
\end{array} \\
& M_{1}\left(v^{1} \cdot x_{o}^{1}\right)-\left(w^{1} \cdot z_{o}^{1}+u^{1} \cdot y_{o}^{1}+u_{0}\right) \leq t_{1}, \\
& \left\|\left[\begin{array}{c}
2 \\
s_{1}-v^{1} \cdot x_{o}^{1}
\end{array}\right]\right\| \leq s_{1}+v^{1} \cdot x_{o}^{1}, \\
& M_{2}\left(w^{1} \cdot z_{o}^{1}+v^{2} \cdot x_{o}^{2}\right)-\left(w^{2} \cdot z_{o}^{2}+u^{2} \cdot y_{o}^{2}+w_{0}\right) \leq t_{2}, \\
& \left\|\left[\begin{array}{c}
2 \\
s_{2}-\left(w^{1} \cdot z_{o}^{1}+v^{2} \cdot x_{o}^{2}\right)
\end{array}\right]\right\| \leq s_{2}+w^{1} \cdot z_{o}^{1}+v^{2} \cdot x_{o}^{2}, \\
& M_{3}\left(w^{2} \cdot z_{o}^{2}+v^{3} \cdot x_{o}^{3}\right)-\left(u^{3} \cdot y_{o}^{3}+v_{0}\right) \leq t_{3}, \\
& \left\|\left[\begin{array}{c}
2 \\
s_{3}-\left(w^{2} \cdot z_{o}^{2}+v^{3} \cdot x_{o}^{3}\right)
\end{array}\right]\right\| \leq s_{3}+w^{2} \cdot z_{o}^{2}+v^{3} \cdot x_{o}^{3}, \\
& Z^{1} w^{1}+Y^{1} u^{1}+u_{0}-X^{1} v^{1} \leq 0 \text {, } \\
& Z^{2} w^{2}+Y^{2} u^{2}+w_{0}-\left(Z^{1} w^{1}+X^{2} v^{2}\right) \leq 0, \\
& Y^{3} u^{3}+v_{0}-\left(Z^{2} w^{2}+X^{3} v^{3}\right) \leq 0, \\
& 0 \leq w^{1} \cdot z_{o}^{1}+u^{1} \cdot y_{o}^{1}+u_{0} \leq 1 \text {, } \\
& 0 \leq v^{1} \cdot x_{o}^{1} \leq 1 \text {, } \\
& 0 \leq w^{2} \cdot z_{o}^{2}+u^{2} \cdot y_{o}^{2}+w_{0} \leq 1, \\
& 0 \leq w^{1} \cdot z_{o}^{1}+v^{2} \cdot x_{o}^{2} \leq 1, \\
& 0 \leq u^{3} \cdot y_{o}^{3}+v_{0} \leq 1, \\
& 0 \leq w^{2} \cdot z_{o}^{2}+v^{3} \cdot x_{o}^{3} \leq 1, \\
& l_{i}^{o} \leq t_{i} \leq u_{i}^{o}, \\
& L_{i}^{o} \leq s_{i} \leq U_{i}^{o}, \\
& v^{1}, v^{2}, v^{3}, u^{1}, u^{2}, u^{3}, w^{1}, w^{2} \geq 0 ; u_{0}, w_{0}, v_{0} \text { are free. } \\
& i=1,2,3, \\
& i=1,2,3 \text {, }
\end{aligned}
$$

Proof. For simplicity, we denote the feasible set of model (2.4) by $\Omega$. At first, we solve the following three optimization problems:

$$
\begin{aligned}
M_{1}=\max & \frac{w^{1} \cdot z_{o}^{1}+u^{1} \cdot y_{o}^{1}+u_{0}}{v^{1} \cdot x_{o}^{1}} \\
\text { s.t. } & \\
& v^{1}, v^{2}, v^{3}, u^{1}, u^{2}, u^{3}, w^{1}, w^{2}, u_{0}, w_{0}, v_{0} \in \Omega, \\
M_{2}=\max & \frac{w^{2} \cdot z_{o}^{2}+u^{2} \cdot y_{o}^{2}+w_{0}}{w^{1} \cdot z_{o}^{1}+v^{2} \cdot x_{o}^{2}}
\end{aligned}
$$

s.t.

$$
v^{1}, v^{2}, v^{3}, u^{1}, u^{2}, u^{3}, w^{1}, w^{2}, u_{0}, w_{0}, v_{0} \in \Omega
$$


and

$$
\begin{aligned}
M_{3}=\max & \frac{u^{3} \cdot y_{o}^{3}+v_{0}}{w^{2} \cdot z_{o}^{2}+v^{3} \cdot x_{o}^{3}} \\
\text { s.t. } & v^{1}, v^{2}, v^{3}, u^{1}, u^{2}, u^{3}, w^{1}, w^{2}, u_{0}, w_{0}, v_{0} \in \Omega .
\end{aligned}
$$

Now, we convert model (2.4) from a maximization problem to a minimization problem:

$$
\begin{aligned}
\min & \alpha\left(\frac{M_{1}\left(v^{1} \cdot x_{o}^{1}\right)-\left(w^{1} \cdot z_{o}^{1}+u^{1} \cdot y_{o}^{1}+u_{0}\right)}{v^{1} \cdot x_{o}^{1}}\right)+\beta\left(\frac{M_{2}\left(w^{1} \cdot z_{o}^{1}+v^{2} \cdot x_{o}^{2}\right)-\left(w^{2} \cdot z_{o}^{2}+u^{2} \cdot y_{o}^{2}+w_{0}\right)}{w^{1} \cdot z_{o}^{1}+v^{2} \cdot x_{o}^{2}}\right) \\
& +\gamma\left(\frac{M_{3}\left(w^{2} \cdot z_{o}^{2}+v^{3} \cdot x_{o}^{3}\right)-\left(u^{3} \cdot y_{o}^{3}+v_{0}\right)}{w^{2} \cdot z_{o}^{2}+v^{3} \cdot x_{o}^{3}}\right)
\end{aligned}
$$

s.t.

$$
v^{1}, v^{2}, v^{3}, u^{1}, u^{2}, u^{3}, w^{1}, w^{2}, u_{0}, w_{0}, v_{0} \in \Omega .
$$

Then, let

$$
\begin{aligned}
M_{1}\left(v^{1} \cdot x_{o}^{1}\right)-\left(w^{1} \cdot z_{o}^{1}+u^{1} \cdot y_{o}^{1}+u_{0}\right) & \leq t_{1}, \\
\frac{1}{v^{1} \cdot x_{o}^{1}} & \leq s_{1}, \\
M_{2}\left(w^{1} \cdot z_{o}^{1}+v^{2} \cdot x_{o}^{2}\right)-\left(w^{2} \cdot z_{o}^{2}+u^{2} \cdot y_{o}^{2}+w_{0}\right) & \leq t_{2}, \\
\frac{1}{w^{1} \cdot z_{o}^{1}+v^{2} \cdot x_{o}^{2}} & \leq s_{2}, \\
M_{3}\left(w^{2} \cdot z_{o}^{2}+v^{3} \cdot x_{o}^{3}\right) & -\left(u^{3} \cdot y_{o}^{3}+v_{0}\right) \leq t_{3}, \\
\frac{1}{w^{2} \cdot z_{o}^{2}+v^{3} \cdot x_{o}^{3}} & \leq s_{3} .
\end{aligned}
$$

In the sequel, to determine intervals containing $t_{i}, i=1,2,3$ and $s_{i}, i=1,2,3$, the following linear programming problems are solved:

$$
\begin{aligned}
\max / \min & M_{1}\left(v^{1} \cdot x_{o}^{1}\right)-\left(w^{1} \cdot z_{o}^{1}+u^{1} \cdot y_{o}^{1}+u_{0}\right) \\
\text { s.t. } & \\
& v^{1}, v^{2}, v^{3}, u^{1}, u^{2}, u^{3}, w^{1}, w^{2}, u_{0}, w_{0}, v_{0} \in \Omega, \\
\max / \min & v^{1} \cdot x_{o}^{1} \\
\text { s.t. } & \\
& v^{1}, v^{2}, v^{3}, u^{1}, u^{2}, u^{3}, w^{1}, w^{2}, u_{0}, w_{0}, v_{0} \in \Omega, \\
\max / \min & M_{2}\left(w^{1} \cdot z_{o}^{1}+v^{2} \cdot x_{o}^{2}\right)-\left(w^{2} \cdot z_{o}^{2}+u^{2} \cdot y_{o}^{2}+w_{0}\right)
\end{aligned}
$$

s.t.

$$
v^{1}, v^{2}, v^{3}, u^{1}, u^{2}, u^{3}, w^{1}, w^{2}, u_{0}, w_{0}, v_{0} \in \Omega,
$$

$\max / \min w^{1} \cdot z_{o}^{1}+v^{2} \cdot x_{o}^{2}$

s.t.

$$
v^{1}, v^{2}, v^{3}, u^{1}, u^{2}, u^{3}, w^{1}, w^{2}, u_{0}, w_{0}, v_{0} \in \Omega,
$$

$\max / \min M_{3}\left(w^{2} \cdot z_{o}^{2}+v^{3} \cdot x_{o}^{3}\right)-\left(u^{3} \cdot y_{o}^{3}+v_{0}\right)$

s.t. 


$$
v^{1}, v^{2}, v^{3}, u^{1}, u^{2}, u^{3}, w^{1}, w^{2}, u_{0}, w_{0}, v_{0} \in \Omega
$$

and

$$
\begin{aligned}
\max / \min & w^{2} \cdot z_{o}^{2}+v^{3} \cdot x_{o}^{3} \\
\text { s.t. } & \\
& v^{1}, v^{2}, v^{3}, u^{1}, u^{2}, u^{3}, w^{1}, w^{2}, u_{0}, w_{0}, v_{0} \in \Omega .
\end{aligned}
$$

Let the minimum and maximum of models (2.10), (2.12) and (2.14) be $l_{i}^{o}, i=1,2,3$ and $u_{i}^{o}, i=1,2,3$, respectively. Likewise, the reciprocal of the maximum and minimum of models (2.11), (2.13) and (2.15) be $L_{i}^{o}, i=1,2,3$ and $U_{i}^{o}, i=1,2,3$, respectively. Then, we have $l_{i}^{o} \leq t_{i} \leq u_{i}^{o}, i=1,2,3$ and $L_{i}^{o} \leq s_{i} \leq U_{i}^{o}, i=1,2,3$. Thus, model (2.9) is equivalent to

$$
\begin{aligned}
& \min \alpha\left(t_{1} s_{1}\right)+\beta\left(t_{2} s_{2}\right)+\gamma\left(t_{3} s_{3}\right) \\
& \text { s.t. } \\
& M_{1}\left(v^{1} \cdot x_{o}^{1}\right)-\left(w^{1} \cdot z_{o}^{1}+u^{1} \cdot y_{o}^{1}+u_{0}\right) \leq t_{1}, \\
& \frac{1}{v^{1} \cdot x_{o}^{1}} \leq s_{1} \\
& M_{2}\left(w^{1} \cdot z_{o}^{1}+v^{2} \cdot x_{o}^{2}\right)-\left(w^{2} \cdot z_{o}^{2}+u^{2} \cdot y_{o}^{2}+w_{0}\right) \leq t_{2}, \\
& \frac{1}{w^{1} \cdot z_{o}^{1}+v^{2} \cdot x_{o}^{2}} \leq s_{2}, \\
& M_{3}\left(w^{2} \cdot z_{o}^{2}+v^{3} \cdot x_{o}^{3}\right)-\left(u^{3} \cdot y_{o}^{3}+v_{0}\right) \leq t_{3}, \\
& \frac{1}{w^{2} \cdot z_{o}^{2}+v^{3} \cdot x_{o}^{3}} \leq s_{3}, \\
& l_{i}^{o} \leq t_{i} \leq u_{i}^{o} \\
& L_{i}^{o} \leq s_{i} \leq U_{i}^{o}, \\
& v^{1}, v^{2}, v^{3}, u^{1}, u^{2}, u^{3}, w^{1}, w^{2}, u_{0}, w_{0}, v_{0} \in \Omega . \\
& i=1,2,3, \\
& i=1,2,3 \text {, }
\end{aligned}
$$

Further, let $\theta_{i}, i=1,2,3$ be such that

$$
t_{i} s_{i} \leq \theta_{i}, \quad i=1,2,3 .
$$

Thus, model (2.16) is equivalent to

$$
\begin{array}{cll}
\min & \alpha \theta_{1}+\beta \theta_{2}+\gamma \theta_{3} & \\
\text { s.t. } & & \\
& t_{i} s_{i} \leq \theta_{i}, & \\
& M_{1}\left(v^{1} \cdot x_{o}^{1}\right)-\left(w^{1} \cdot z_{o}^{1}+u^{1} \cdot y_{o}^{1}+u_{0}\right) \leq t_{1}, 2,3, \\
& 1 \leq s_{1}\left(v^{1} \cdot x_{o}^{1}\right), & \\
& M_{2}\left(w^{1} \cdot z_{o}^{1}+v^{2} \cdot x_{o}^{2}\right)-\left(w^{2} \cdot z_{o}^{2}+u^{2} \cdot y_{o}^{2}+w_{0}\right) \leq t_{2}, & \\
& 1 \leq s_{2}\left(w^{1} \cdot z_{o}^{1}+v^{2} \cdot x_{o}^{2}\right), & \\
& M_{3}\left(w^{2} \cdot z_{o}^{2}+v^{3} \cdot x_{o}^{3}\right)-\left(u^{3} \cdot y_{o}^{3}+v_{0}\right) \leq t_{3}, & \\
& 1 \leq s_{3}\left(w^{2} \cdot z_{o}^{2}+v^{3} \cdot x_{o}^{3}\right), & i=1,2,3, \\
& l_{i}^{o} \leq t_{i} \leq u_{i}^{o}, & i=1,2,3, \\
& L_{i}^{o} \leq s_{i} \leq U_{i}^{o}, &
\end{array}
$$




$$
v^{1}, v^{2}, v^{3}, u^{1}, u^{2}, u^{3}, w^{1}, w^{2}, u_{0}, w_{0}, v_{0} \in \Omega
$$

Since

$$
\begin{aligned}
\left(u_{i}^{o}-t_{i}\right)\left(U_{i}^{o}-s_{i}\right)=u_{i}^{o} U_{i}^{o}-u_{i}^{o} s_{i}-t_{i} U_{i}^{o}+t_{i} s_{i} \geq 0, & i=1,2,3 \\
\left(t_{i}-l_{i}^{o}\right)\left(s_{i}-L_{i}^{o}\right)=t_{i} s_{i}-t_{i} L_{i}^{o}-l_{i}^{o} s_{i}+l_{i}^{o} L_{i}^{o} \geq 0, & i=1,2,3 .
\end{aligned}
$$

Then

$$
\begin{array}{ll}
t_{i} s_{i} \geq u_{i}^{o} s_{i}+t_{i} U_{i}^{o}-u_{i}^{o} U_{i}^{o}, & i=1,2,3 \\
t_{i} s_{i} \geq t_{i} L_{i}^{o}+l_{i}^{o} s_{i}-l_{i}^{o} L_{i}^{o}, & i=1,2,3 .
\end{array}
$$

Using these inequalities, the first set of constraints are relaxed. Further, using Lemma 2.1, the third, fifth and seventh constraints can be presented as conic forms. Thus the relaxed form of (2.17) becomes:

$$
\begin{array}{ll}
\min & \alpha \theta_{1}+\beta \theta_{2}+\gamma \theta_{3} \\
\text { s.t. } & \\
& \max \left\{u_{i}^{o} s_{i}+t_{i} U_{i}^{o}-u_{i}^{o} U_{i}^{o}, t_{i} L_{i}^{o}+l_{i}^{o} s_{i}-l_{i}^{o} L_{i}^{o}\right\} \leq \theta_{i}, \quad i=1,2,3, \\
& M_{1}\left(v^{1} \cdot x_{o}^{1}\right)-\left(w^{1} \cdot z_{o}^{1}+u^{1} \cdot y_{o}^{1}+u_{0}\right) \leq t_{1}, \\
& \left\|\left[\begin{array}{l}
2 \\
s_{1}-v^{1} \cdot x_{o}^{1}
\end{array}\right]\right\| \leq s_{1}+v^{1} \cdot x_{o}^{1}, \\
& M_{2}\left(w^{1} \cdot z_{o}^{1}+v^{2} \cdot x_{o}^{2}\right)-\left(w^{2} \cdot z_{o}^{2}+u^{2} \cdot y_{o}^{2}+w_{0}\right) \leq t_{2}, \\
& \|\left[\begin{array}{l}
2 \\
\end{array}\right. \\
& M_{3}\left(w_{2}-\left(w^{1} \cdot z_{o}^{2}+v^{3} \cdot v^{2} \cdot x_{o}^{2}\right)\right] \| \leq s_{2}+w^{1} \cdot z_{o}^{1}+v^{2} \cdot x_{o}^{2}, \\
& \|\left[u^{3} \cdot y_{o}^{3}+v_{0}\right) \leq t_{3}, \\
& \left.\| s_{3}-\left(w^{2} \cdot z_{o}^{2}+v^{3} \cdot x_{o}^{3}\right)\right] \| \leq s_{3}+w^{2} \cdot z_{o}^{2}+v^{3} \cdot x_{o}^{3}, \\
& l_{i}^{o} \leq t_{i} \leq u_{i}^{o}, \\
& L_{i}^{o} \leq s_{i} \leq U_{i}^{o}, \\
& v^{1}, v^{2}, v^{3}, u^{1}, u^{2}, u^{3}, w^{1}, w^{2}, u_{0}, w_{0}, v_{0} \in \Omega .
\end{array}
$$

Thus, we get model (2.5).

The MED is the other approach for decomposing the overall efficiency in the network structures that has been used in $[1,42]$ for different multi-stage structures. The MED model for the overall efficiency of the structure as given in Figure 1 is as follows:

$$
\begin{aligned}
E_{o}^{\mathrm{MC}}=\max & \frac{w^{1} \cdot z_{o}^{1}+u^{1} \cdot y_{o}^{1}+u_{0}}{v^{1} \cdot x_{o}^{1}} \times \frac{w^{2} \cdot z_{o}^{2}+u^{2} \cdot y_{o}^{2}+w_{0}}{w^{1} \cdot z_{o}^{1}+v^{2} \cdot x_{o}^{2}} \times \frac{u^{3} \cdot y_{o}^{3}+v_{0}}{w^{2} \cdot z_{o}^{2}+v^{3} \cdot x_{o}^{3}} \\
\text { s.t. } & \frac{Z^{1} w^{1}+Y^{1} u^{1}+u_{0}}{X^{1} v^{1}} \leq 1, \\
& \frac{Z^{2} w^{2}+Y^{2} u^{2}+w_{0}}{Z^{1} w^{1}+X^{2} v^{2}} \leq 1, \\
& \frac{Y^{3} u^{3}+v_{0}}{Z^{2} w^{2}+X^{3} v^{3}} \leq 1, \\
& v^{1}, v^{2}, v^{3}, u^{1}, u^{2}, u^{3}, w^{1}, w^{2} \geq 0 ; u_{0}, w_{0}, v_{0} \text { are free. }
\end{aligned}
$$


Model (2.18) is also a fractional nonlinear programming problem that cannot be linearized using CharnesCooper tansformation. In the following theorem, like Theorem 2.2, a conic relaxation of model (2.18) is given. First, consider (2.18) with extra constraints (2.3) as follows:

$$
\begin{array}{ll}
\min & \frac{v^{1} \cdot x_{o}^{1}}{w^{1} \cdot z_{o}^{1}+u^{1} \cdot y_{o}^{1}+u_{0}} \times \frac{w^{1} \cdot z_{o}^{1}+v^{2} \cdot x_{o}^{2}}{w^{2} \cdot z_{o}^{2}+u^{2} \cdot y_{o}^{2}+w_{0}} \times \frac{w^{2} \cdot z_{o}^{2}+v^{3} \cdot x_{o}^{3}}{u^{3} \cdot y_{o}^{3}+v_{0}} \\
\text { s.t. } & \\
& \frac{Z^{1} w^{1}+Y^{1} u^{1}+u_{0}}{X^{1} v^{1}} \leq 1 \\
& \frac{Z^{2} w^{2}+Y^{2} u^{2}+w_{0}}{Z^{1} w^{1}+X^{2} v^{2}} \leq 1 \\
& \frac{Y^{3} u^{3}+v_{0}}{Z^{2} w^{2}+X^{3} v^{3}} \leq 1 \\
& 0 \leq w^{1} \cdot z_{o}^{1}+u^{1} \cdot y_{o}^{1}+u_{0} \leq 1 \\
& 0 \leq v^{1} \cdot x_{o}^{1} \leq 1 \\
& 0 \leq w^{2} \cdot z_{o}^{2}+u^{2} \cdot y_{o}^{2}+w_{0} \leq 1 \\
& 0 \leq w^{1} \cdot z_{o}^{1}+v^{2} \cdot x_{o}^{2} \leq 1 \\
& 0 \leq u^{3} \cdot y_{o}^{3}+v_{0} \leq 1 \\
& 0 \leq w^{2} \cdot z_{o}^{2}+v^{3} \cdot x_{o}^{3} \leq 1 \\
& v^{1}, v^{2}, v^{3}, u^{1}, u^{2}, u^{3}, w^{1}, w^{2} \geq 0 ; u_{0}, w_{0}, v_{0} \text { are free. }
\end{array}
$$

Theorem 2.3. Conic relaxation of model (2.19) is as follows:

$$
\begin{aligned}
& E_{o}^{\mathrm{MC}}=\min \beta \\
& \text { s.t. } \\
& \theta_{1}+\theta_{2}+\theta_{3}-2 \leq \beta, \\
& u_{i}^{o} s_{i}+t_{i} U_{i}^{o}-u_{i}^{o} U_{i}^{o} \leq \theta_{i}, \\
& t_{i} L_{i}^{o}+l_{i}^{o} s_{i}-l_{i}^{o} L_{i}^{o} \leq \theta_{i}, \\
& v^{1} \cdot x_{o}^{1} \leq t_{1}, \\
& \left\|\left[\begin{array}{c}
2 \\
s_{1}-\left(w^{1} \cdot z_{o}^{1}+u^{1} \cdot y_{o}^{1}+u_{0}\right)
\end{array}\right]\right\| \leq s_{1}+w^{1} \cdot z_{o}^{1}+u^{1} \cdot y_{o}^{1}+u_{0}, \\
& w^{1} \cdot z_{o}^{1}+v^{2} \cdot x_{o}^{2} \leq t_{2}, \\
& \left\|\left[\begin{array}{c}
2 \\
s_{2}-\left(w^{2} \cdot z_{o}^{2}+u^{2} \cdot y_{o}^{2}+w_{0}\right)
\end{array}\right]\right\| \leq s_{2}+w^{2} \cdot z_{o}^{2}+u^{2} \cdot y_{o}^{2}+w_{0}, \\
& w^{2} \cdot z_{o}^{2}+v^{3} \cdot x_{o}^{3} \leq t_{3}, \\
& \left\|\left[\begin{array}{c}
2 \\
s_{3}-\left(u^{3} \cdot y_{o}^{3}+v_{0}\right)
\end{array}\right]\right\| \leq s_{3}+u^{3} \cdot y_{o}^{3}+v_{0} \\
& Z^{1} w^{1}+Y^{1} u^{1}+u_{0}-X^{1} v^{1} \leq 0, \\
& Z^{2} w^{2}+Y^{2} u^{2}+w_{0}-\left(Z^{1} w^{1}+X^{2} v^{2}\right) \leq 0, \\
& Y^{3} u^{3}+v_{0}-\left(Z^{2} w^{2}+X^{3} v^{3}\right) \leq 0, \\
& 0 \leq w^{1} \cdot z_{o}^{1}+u^{1} \cdot y_{o}^{1}+u_{0} \leq 1 \text {, } \\
& 0 \leq v^{1} \cdot x_{o}^{1} \leq 1 \text {, } \\
& i=1,2,3 \text {, } \\
& i=1,2,3 \text {, }
\end{aligned}
$$




$$
\begin{array}{ll}
0 \leq w^{2} \cdot z_{o}^{2}+u^{2} \cdot y_{o}^{2}+w_{0} \leq 1, & \\
0 \leq w^{1} \cdot z_{o}^{1}+v^{2} \cdot x_{o}^{2} \leq 1, & \\
0 \leq u^{3} \cdot y_{o}^{3}+v_{0} \leq 1, & \\
0 \leq w^{2} \cdot z_{o}^{2}+v^{3} \cdot x_{o}^{3} \leq 1, & i=1,2,3, \\
l_{i}^{o} \leq t_{i} \leq u_{i}^{o}, & i=1,2,3, \\
L_{i}^{o} \leq s_{i} \leq U_{i}^{o}, & \\
v^{1}, v^{2}, v^{3}, u^{1}, u^{2}, u^{3}, w^{1}, w^{2} \geq 0 ; u_{0}, w_{0}, v_{0} \text { are free. } &
\end{array}
$$

Proof. For simplicity, we denote the feasible set of model (2.19) by $\Psi$. Let

$$
\begin{aligned}
v^{1} \cdot x_{o}^{1} & \leq t_{1}, \\
\frac{1}{w^{1} \cdot z_{o}^{1}+u^{1} \cdot y_{o}^{1}+u_{0}} & \leq s_{1}, \\
w^{1} \cdot z_{o}^{1}+v^{2} \cdot x_{o}^{2} & \leq t_{2}, \\
\frac{1}{w^{2} \cdot z_{o}^{2}+u^{2} \cdot y_{o}^{2}+w_{0}} & \leq s_{2}, \\
w^{2} \cdot z_{o}^{2}+v^{3} \cdot x_{o}^{3} & \leq t_{3}, \\
\frac{1}{u^{3} \cdot y_{o}^{3}+v_{0}} & \leq s_{3} .
\end{aligned}
$$

In the sequel, to determine intervals containing $t_{i}, i=1,2,3$ and $s_{i}, i=1,2,3$, the following linear programming problems are solved:

$$
\begin{aligned}
\max / \min & v^{1} \cdot x_{o}^{1} \\
\text { s.t. } & \\
& v^{1}, v^{2}, v^{3}, u^{1}, u^{2}, u^{3}, w^{1}, w^{2}, u_{0}, w_{0}, v_{0} \in \Psi, \\
\max / \min & w^{1} \cdot z_{o}^{1}+u^{1} \cdot y_{o}^{1}+u_{0}
\end{aligned}
$$

s.t.

$$
\begin{aligned}
& v^{1}, v^{2}, v^{3}, u^{1}, u^{2}, u^{3}, w^{1}, w^{2}, u_{0}, w_{0}, v_{0} \in \Psi, \\
& \max / \min w^{1} \cdot z_{o}^{1}+v^{2} \cdot x_{o}^{2}
\end{aligned}
$$

s.t.

$$
v^{1}, v^{2}, v^{3}, u^{1}, u^{2}, u^{3}, w^{1}, w^{2}, u_{0}, w_{0}, v_{0} \in \Psi,
$$

$\max / \min w^{2} \cdot z_{o}^{2}+u^{2} \cdot y_{o}^{2}+w_{0}$

s.t.

$$
v^{1}, v^{2}, v^{3}, u^{1}, u^{2}, u^{3}, w^{1}, w^{2}, u_{0}, w_{0}, v_{0} \in \Psi
$$
$\max / \min w^{2} \cdot z_{o}^{2}+v^{3} \cdot x_{o}^{3}$

s.t.

$$
v^{1}, v^{2}, v^{3}, u^{1}, u^{2}, u^{3}, w^{1}, w^{2}, u_{0}, w_{0}, v_{0} \in \Psi
$$

and

$$
\begin{aligned}
\max / \min & u^{3} \cdot y_{o}^{3}+v_{0} \\
\text { s.t. } & \\
& v^{1}, v^{2}, v^{3}, u^{1}, u^{2}, u^{3}, w^{1}, w^{2}, u_{0}, w_{0}, v_{0} \in \Psi .
\end{aligned}
$$


Let the minimum and maximum of models $(2.22),(2.24)$ and $(2.26)$ be $l_{i}^{o}, i=1,2,3$ and $u_{i}^{o}, i=1,2,3$, respectively. Likewise, the reciprocal of the maximum and minimum of models (2.23), (2.25) and (2.27) be $L_{i}^{o}, i=1,2,3$ and $U_{i}^{o}, i=1,2,3$, respectively. Then, we have $l_{i}^{o} \leq t_{i} \leq u_{i}^{o}, i=1,2,3$ and $L_{i}^{o} \leq s_{i} \leq U_{i}^{o}, i=1,2,3$. Thus, model (2.19) is equivalent to

$$
\begin{aligned}
& \min \left(t_{1} s_{1}\right) \times\left(t_{2} s_{2}\right) \times\left(t_{3} s_{3}\right) \\
& \text { s.t. } \\
& v^{1} \cdot x_{o}^{1} \leq t_{1}, \\
& \frac{1}{w^{1} \cdot z_{o}^{1}+u^{1} \cdot y_{o}^{1}+u_{0}} \leq s_{1}, \\
& w^{1} \cdot z_{o}^{1}+v^{2} \cdot x_{o}^{2} \leq t_{2}, \\
& \frac{1}{w^{2} \cdot z_{o}^{2}+u^{2} \cdot y_{o}^{2}+w_{0}} \leq s_{2}, \\
& w^{2} \cdot z_{o}^{2}+v^{3} \cdot x_{o}^{3} \leq t_{3}, \\
& \frac{1}{u^{3} \cdot y_{o}^{3}+v_{0}} \leq s_{3}, \\
& l_{i}^{o} \leq t_{i} \leq u_{i}^{o}, \\
& L_{i}^{o} \leq s_{i} \leq U_{i}^{o} \text {, } \\
& v^{1}, v^{2}, v^{3}, u^{1}, u^{2}, u^{3}, w^{1}, w^{2}, u_{0}, w_{0}, v_{0} \in \Psi \text {. } \\
& \begin{array}{l}
i=1,2,3, \\
i=1,2,3,
\end{array}
\end{aligned}
$$

Now, let $\theta_{i}, i=1,2,3$ and $\beta$ be such that

$$
\begin{aligned}
t_{i} s_{i} & \leq \theta_{i}, \quad i=1,2,3, \\
\theta_{1} \theta_{2} \theta_{3} & \leq \beta .
\end{aligned}
$$

Thus, model (2.28) is equivalent to

$$
\begin{aligned}
& \min \beta \\
& \text { s.t. } \\
& \theta_{1} \theta_{2} \theta_{3} \leq \beta, \\
& t_{i} s_{i} \leq \theta_{i}, i=1,2,3, \\
& v^{1} \cdot x_{o}^{1} \leq t_{1}, \\
& 1 \leq s_{1}\left(w^{1} \cdot z_{o}^{1}+u^{1} \cdot y_{o}^{1}+u_{0}\right), \\
& w^{1} \cdot z_{o}^{1}+v^{2} \cdot x_{o}^{2} \leq t_{2}, \\
& 1 \leq s_{2}\left(w^{2} \cdot z_{o}^{2}+u^{2} \cdot y_{o}^{2}+w_{0}\right), \\
& w^{2} \cdot z_{o}^{2}+v^{3} \cdot x_{o}^{3} \leq t_{3}, \\
& 1 \leq s_{3}\left(u^{3} \cdot y_{o}^{3}+v_{0}\right), \\
& l_{i}^{o} \leq t_{i} \leq u_{i}^{o}, \\
& i=1,2,3, \\
& L_{i}^{o} \leq s_{i} \leq U_{i}^{o}, \\
& i=1,2,3 \text {, } \\
& v^{1}, v^{2}, v^{3}, u^{1}, u^{2}, u^{3}, w^{1}, w^{2}, u_{0}, w_{0}, v_{0} \in \Psi \text {. }
\end{aligned}
$$

Further, we know that

$$
\theta_{1}+\theta_{2}+\theta_{3}-2 \leq \theta_{1} \theta_{2} \theta_{3} .
$$


Similar to the AED case

$$
\begin{aligned}
\left(u_{i}^{o}-t_{i}\right)\left(U_{i}^{o}-s_{i}\right)=u_{i}^{o} U_{i}^{o}-u_{i}^{o} s_{i}-t_{i} U_{i}^{o}+t_{i} s_{i} \geq 0, & i=1,2,3 \\
\left(t_{i}-l_{i}^{o}\right)\left(s_{i}-L_{i}^{o}\right)=t_{i} s_{i}-t_{i} L_{i}^{o}-l_{i}^{o} s_{i}+l_{i}^{o} L_{i}^{o} \geq 0, & i=1,2,3,
\end{aligned}
$$

then

$$
\begin{array}{ll}
t_{i} s_{i} \geq u_{i}^{o} s_{i}+t_{i} U_{i}^{o}-u_{i}^{o} U_{i}^{o}, & i=1,2,3 \\
t_{i} s_{i} \geq t_{i} L_{i}^{o}+l_{i}^{o} s_{i}-l_{i}^{o} L_{i}^{o}, & i=1,2,3 .
\end{array}
$$

Using these inequalities, the first and second set of constraints are relaxed. Furthur, using Lemma 2.1, the fourth, sixth and eighth constraints can be presented as conic forms. Thus, the relaxed form of (2.29) becomes:

$$
\begin{aligned}
& \min \beta \\
& \text { s.t. } \\
& \theta_{1}+\theta_{2}+\theta_{3}-2 \leq \beta, \\
& \max \left\{u_{i}^{o} s_{i},+t_{i} U_{i}^{o}-u_{i}^{o} U_{i}^{o}, t_{i} L_{i}^{o}+l_{i}^{o} s_{i}-l_{i}^{o} L_{i}^{o}\right\} \leq \theta_{i}, \quad i=1,2,3, \\
& v^{1} \cdot x_{o}^{1} \leq t_{1}, \\
& \left\|\left[\begin{array}{c}
2 \\
s_{1}-\left(w^{1} \cdot z_{o}^{1}+u^{1} \cdot y_{o}^{1}+u_{0}\right)
\end{array}\right]\right\| \leq s_{1}+w^{1} \cdot z_{o}^{1}+u^{1} \cdot y_{o}^{1}+u_{0}, \\
& w^{1} \cdot z_{o}^{1}+v^{2} \cdot x_{o}^{2} \leq t_{2}, \\
& \left\|\left[\begin{array}{c}
2 \\
s_{2}-\left(w^{2} \cdot z_{o}^{2}+u^{2} \cdot y_{o}^{2}+w_{0}\right)
\end{array}\right]\right\| \leq s_{2}+w^{2} \cdot z_{o}^{2}+u^{2} \cdot y_{o}^{2}+w_{0}, \\
& w^{2} \cdot z_{o}^{2}+v^{3} \cdot x_{o}^{3} \leq t_{3}, \\
& \left\|\left[\begin{array}{c}
2 \\
s_{3}-\left(u^{3} \cdot y_{o}^{3}+v_{0}\right)
\end{array}\right]\right\| \leq s_{3}+u^{3} \cdot y_{o}^{3}+v_{0}, \\
& l_{i}^{o} \leq t_{i} \leq u_{i}^{o} \\
& L_{i}^{o} \leq s_{i} \leq U_{i}^{o}, \\
& v^{1}, v^{2}, v^{3}, u^{1}, u^{2}, u^{3}, w^{1}, w^{2}, u_{0}, w_{0}, v_{0} \in \Psi . \\
& i=1,2,3 \text {, }
\end{aligned}
$$

Thus, we get model (2.20).

Now DMU 's efficiencies of stage 1 , stage 2 and stage 3 using an optimal weight $\left(v^{1 *}, v^{2 *}, u^{1 *}, u^{2 *}, w^{1 *}, w^{2 *}, u_{0}^{*}, w_{0}^{*}, v_{0}^{*}\right)$ of model (2.5) (model $\left.\quad(2.20)\right), \quad$ are calculated as following, respectively:

$$
\begin{aligned}
& E_{o}^{1}=\frac{w^{1 *} \cdot z_{o}^{1}+u^{1 *} \cdot y_{o}^{1}+u_{0}^{*}}{v^{1 *} \cdot x_{o}^{1}}, \\
& E_{o}^{2}=\frac{w^{2 *} \cdot z_{o}^{2}+u^{2 *} \cdot y_{o}^{2}+w_{0}^{*}}{w^{1 *} \cdot z_{o}^{1}+v^{2 *} \cdot x_{o}^{2}}, \\
& E_{o}^{3}=\frac{u^{3 *} \cdot y_{o}^{3}+v_{0}^{*}}{w^{2 *} \cdot z_{o}^{2}+v^{3 *} \cdot x_{o}^{3}} .
\end{aligned}
$$

Thus, the overall efficiency score of the three-stage process in additive model (2.5) becomes $E_{o}^{\mathrm{AC}}=$ $\frac{1}{3}\left(E_{o}^{1}+E_{o}^{2}+E_{o}^{3}\right)$ and in multiplicative model $(2.20)$ becomes $E_{o}^{\mathrm{MC}}=E_{o}^{1} \times E_{o}^{2} \times E_{o}^{3}$. On the other hand, existence of multiple optimal weights in the cooperative models (2.5) and (2.20) may lead to non-unique efficiency decomposition which is the subject of the next section. 


\section{EFFICIENCY DECOMPOSITION BASED ON BARGAINING GAME}

Although cooperative models determine stages' efficiencies and the overall efficiency with common weights and their aim is to maximize the efficiency of system, the overall efficiency derived from these models may not be unique, and thus the stages' efficiencies may also be non-unique [29]. On the other hand, each stage in the cooperative approach tries to achieve weights to maximize its efficiency but cannot effect the weights of the other stages. In other words, each stage's weights are independent of the weights of the other stages, while there exist structures where stages' weights may depend on the weights of other stages [20]. To overcome this issue, we utilize Nash bargaining game approach that has been used in several network DEA models [31,39,45]. First, let us briefly discuss this approach. The set of three persons in the bargaining are shown by $N=\{1,2,3\}$, and a payoff vector is an element of the payoff space $R^{3}$, which is the three-dimensional Euclidean space. A feasible set $S$ and a breakdown point $\vec{b}$ are a subset of the payoff space and an element of the payoff space, respectively. Then a bargaining problem is the triple $(N, S, \vec{b})$ consisting of persons, feasible set, and breakdown point. Nash approach requires the convexity and compactness of the feasible set, furher it should contain some payoff vector such that each person's payoff is at least as large as the person's breakdown payoff. Also, Nash [33,34] argued that a solution should satisfy the following four properties: Pareto efficiency, invariance with respect to affine transformation, independence of irrelevant alternatives, and symmetry. After all, he showed that there always exists a unique solution, called the Nash solution, which satisfies the abovementioned four properties and can be obtaind by solving the following problem:

$$
\max _{\vec{u} \in S, \vec{u} \geq \vec{b}} \prod_{i=1}^{N}\left(u_{i}-b_{i}\right)
$$

where $\vec{u}$ is the payment vector for the persons, and $u_{i}$ and $b_{i}$ are the $i$ th elements of $\vec{u}$ and $\vec{b}$, respectively. In the proposed structure, we consider the stages, the efficiency of each stage and the minimum achievable efficiencies for the stages respectively, as players, the payoff function and the breakdown points. To begin bargaining between stages, we obtain a breakdown point for each stage. In the process, one would not bargain with the other stage if its efficiency is less than the minimum efficiency, else the efficiency of the whole system would decrease. In fact, the benefit is distributed between the players based on their competition and bargaining power. To estimate the breakdown points, we apply the approach of Du et al. [16] that the minimum achievable efficiency for each stage can be obtained by adding a virtual DMU such that $x_{i}^{\max }=\max _{j}\left\{x_{i j}\right\}$ and $y_{r}^{\min }=\min _{j}\left\{y_{r j}\right\}$ for each stage. Since each DMU uses maximum amount of inputs to produce the minimum amount of outputs, efficiency scores calculated by DEA for $\left(x_{i}^{\max }, y_{r}^{\min }\right)$ represents the least achievable efficiency for each stage. Thus, we can formulate the following Nash bargaining game model for measuring efficincy decomposition:

$$
\begin{aligned}
E_{o}^{\mathrm{NC}}=\max & \left(\frac{w^{1} \cdot z_{o}^{1}+u^{1} \cdot y_{o}^{1}+u_{0}}{v^{1} \cdot x_{o}^{1}}-E_{\min }^{1}\right) \times\left(\frac{w^{2} \cdot z_{o}^{2}+u^{2} \cdot y_{o}^{2}+w_{0}}{w^{1} \cdot z_{o}^{1}+v^{2} \cdot x_{o}^{2}}-E_{\min }^{2}\right) \\
& \times\left(\frac{u^{3} \cdot y_{o}^{3}+v_{0}}{w^{2} \cdot z_{o}^{2}+v^{3} \cdot x_{o}^{3}}-E_{\min }^{3}\right)
\end{aligned}
$$

s.t.

$$
\begin{aligned}
& \frac{w^{1} \cdot z_{o}^{1}+u^{1} \cdot y_{o}^{1}+u_{0}}{v^{1} \cdot x_{o}^{1}} \geq E_{\min }^{1} \\
& \frac{w^{2} \cdot z_{o}^{2}+u^{2} \cdot y_{o}^{2}+w_{0}}{w^{1} \cdot z_{o}^{1}+v^{2} \cdot x_{o}^{2}} \geq E_{\min }^{2} \\
& \frac{u^{3} \cdot y_{o}^{3}+v_{0}}{w^{2} \cdot z_{o}^{2}+v^{3} \cdot x_{o}^{3}} \geq E_{\min }^{3} \\
& \frac{Z^{1} w^{1}+Y^{1} u^{1}+u_{0}}{X^{1} v^{1}} \leq 1
\end{aligned}
$$




$$
\begin{aligned}
& \frac{Z^{2} w^{2}+Y^{2} u^{2}+w_{0}}{Z^{1} w^{1}+X^{2} v^{2}} \leq 1, \\
& \frac{Y^{3} u^{3}+v_{0}}{Z^{2} w^{2}+X^{3} v^{3}} \leq 1, \\
& v^{1}, v^{2}, v^{3}, u^{1}, u^{2}, u^{3}, w^{1}, w^{2} \geq 0 ; u_{0}, w_{0}, v_{0} \text { are free }
\end{aligned}
$$

where $E_{\min }^{1}, E_{\min }^{2}$ and $E_{\min }^{3}$ are the minimum efficiencies of stages.

Lemma 3.1. The feasible set of model (3.1) is compact and convex.

Proof. The proof is similar to the proof of Lemma 3.1 in [1].

In the sequel, we give a conic relaxation of the bargaining model (3.1).

Theorem 3.2. Conic relaxation of model (3.1) with extra constraints (2.3) is as follows:

$$
\begin{array}{rlr}
E_{o}^{\mathrm{NC}}=\min & \beta & \\
\text { s.t. } & & \\
& \theta_{1}+\theta_{2}+\theta_{3}-2 \leq \beta, & \\
& u_{i}^{o} s_{i}+t_{i} U_{i}^{o}-u_{i}^{o} U_{i}^{o} \leq \theta_{i}, & i=1,2,3, \\
& t_{i} L_{i}^{o}+l_{i}^{o} s_{i}-l_{i}^{o} L_{i}^{o} \leq \theta_{i}, & \\
& v^{1} \cdot x_{o}^{1} \leq t_{1}, & \\
& \left\|\left[\begin{array}{c}
2 \\
s_{1}-M_{1}
\end{array}\right]\right\| \leq s_{1}+M_{1}, 2,3, \\
& w^{1} \cdot z_{o}^{1}+v^{2} \cdot x_{o}^{2} \leq t_{2}, & \\
& \left\|\left[\begin{array}{c}
2 \\
s_{2}-M_{2}
\end{array}\right]\right\| \leq s_{2}+M_{2}, & \\
& w^{2} \cdot z_{o}^{2}+v^{3} \cdot x_{o}^{3} \leq t_{3}, & \\
& \left\|\left[\begin{array}{c}
2 \\
s_{3}-M_{3}
\end{array}\right]\right\| \leq s_{3}+M_{3}, & \\
& l_{i}^{o} \leq t_{i} \leq u_{i}^{o}, & \\
& L_{i}^{o} \leq s_{i} \leq U_{i}^{o}, & \\
& v^{1}, v^{2}, v^{3}, u^{1}, u^{2}, u^{3}, w^{1}, w^{2}, u_{0}, w_{0}, v_{0} \in \Omega=1,2,3,3,
\end{array}
$$

where

$$
\begin{aligned}
& M_{1}=w^{1} \cdot z_{o}^{1}+u^{1} \cdot y_{o}^{1}+u_{0}-E_{\min }^{1}\left(v^{1} \cdot x_{o}^{1}\right), \\
& M_{2}=w^{2} \cdot z_{o}^{2}+u^{2} \cdot y_{o}^{2}+w_{0}-E_{\min }^{2}\left(v^{2} \cdot x_{o}^{2}+w^{1} \cdot z_{o}^{1}\right), \\
& M_{3}=u^{3} \cdot y_{o}^{3}+v_{0}-E_{\min }^{3}\left(v^{3} \cdot x_{o}^{3}+w^{2} \cdot z_{o}^{2}\right) .
\end{aligned}
$$

Proof. The proof is similar to the proof of Theorem 2.3.

Now the stages' efficiency scores that are unique can be computed as the follows:

$$
\begin{aligned}
& E_{o}^{1}=\frac{w^{1 *} \cdot z_{o}^{1}+u^{1 *} \cdot y_{o}^{1}+u_{0}^{*}}{v^{1 *} \cdot x_{o}^{1}}, \\
& E_{o}^{2}=\frac{w^{2 *} \cdot z_{o}^{2}+u^{2 *} \cdot y_{o}^{2}+w_{0}^{*}}{w^{1 *} \cdot z_{o}^{1}+v^{2 *} \cdot x_{o}^{2}},
\end{aligned}
$$




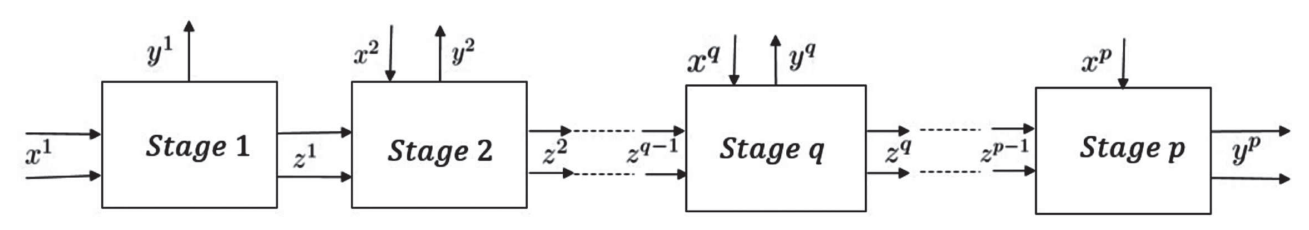

Figure 2. General multi-stage series structure.

$$
E_{o}^{3}=\frac{u^{3 *} \cdot y_{o}^{3}+v_{0}^{*}}{w^{2 *} \cdot z_{o}^{2}+v^{3 *} \cdot x_{o}^{3}},
$$

where $\left(v^{1 *}, v^{2 *}, v^{3 *}, u^{1 *}, u^{2 *}, u^{3 *}, w^{1 *}, w^{2 *}, u_{0}^{*}, w_{0}^{*}, v_{0}^{*}\right)$ are the optimal weights of model (3.2).

\section{General multi-Stage structure}

In this section, we consider a general multi-stage series peocess, as depicted in Figure 2 [14]. The overall efficiency based on Nash bargaining game approach is obtained by the following model:

$$
\begin{aligned}
E_{o}^{\mathrm{GNC}}=\max & \left(\frac{w^{1} \cdot z_{o}^{1}+u^{1} \cdot y_{o}^{1}+w_{0}^{1}}{v^{1} \cdot x_{o}^{1}}-E_{\min }^{1}\right) \prod_{q=2}^{p-1}\left(\frac{w^{q} \cdot z_{o}^{q}+u^{q} \cdot y_{o}^{q}+w_{0}^{q}}{w^{q-1} \cdot z_{o}^{q-1}+v^{q} \cdot x_{o}^{q}}-E_{\min }^{q}\right) \\
& \left(\frac{u^{p} \cdot y_{o}^{p}+w_{0}^{p}}{w^{p-1} \cdot z_{o}^{p-1}+v^{p} \cdot x_{o}^{p}}-E_{\min }^{p}\right)
\end{aligned}
$$

s.t.

$$
\begin{aligned}
& \frac{w^{1} \cdot z_{o}^{1}+u^{1} \cdot y_{o}^{1}+w_{0}^{1}}{v^{1} \cdot x_{o}^{1}} \geq E_{\min }^{1}, \\
& \frac{w^{q} \cdot z_{o}^{q}+u^{q} \cdot y_{o}^{q}+w_{0}^{q}}{w^{q-1} \cdot z_{o}^{q-1}+v^{q} \cdot x_{o}^{q}} \geq E_{\min }^{q}, \\
& \frac{u^{p} \cdot y_{o}^{p}+w_{0}^{p}}{w^{p-1} \cdot z_{o}^{p-1}+v^{p} \cdot x_{o}^{p}} \geq E_{\min }^{p}, \\
& \frac{Z^{1} w^{1}+Y^{1} u^{1}+w_{0}^{1}}{X^{1} v^{1}} \leq 1, \\
& \frac{Z^{q} w^{q}+Y^{q} u^{q}+w_{0}^{q}}{Z^{q-1} w^{q-1}+X^{q} v^{q}} \leq 1, \\
& \frac{Y^{p} u^{p}+w_{0}^{p}}{Z^{p-1} w^{p-1}+X^{p} v^{p}} \leq 1, \\
& v^{q}, u^{q}, w^{q} \geq 0
\end{aligned}
$$$$
q=2, \ldots, p-1
$$$$
q=2, \ldots, p-1,
$$$$
q=1, \ldots, p ; w_{0}^{q} \text { are free. }
$$

Theorem 4.1. Let $p$ be number of stages. Then the conic relaxation of model (4.1) with extra constraints (2.3) is as follows:

$\min \beta$

s.t.

$$
\begin{aligned}
& \sum_{q=1}^{p} \theta_{q}-(p-1) \leq \beta, \\
& u_{q}^{o} s_{q}+t_{q} U_{q}^{o}-u_{q}^{o} U_{q}^{o} \leq \theta_{q}, \quad q=1,2, \ldots, p,
\end{aligned}
$$




$$
\begin{array}{ll}
t_{q} L_{q}^{o}+l_{q}^{o} s_{q}-l_{q}^{o} L_{q}^{o} \leq \theta_{q}, & q=1,2, \ldots, p, \\
v^{1} \cdot x_{o}^{1} \leq t_{1}, & \\
\left\|\left[\begin{array}{c}
2 \\
s_{1}-M_{1}
\end{array}\right]\right\| \leq s_{1}+M_{1}, & \\
w^{q-1} \cdot z_{o}^{q-1}+v^{q} \cdot x_{o}^{q} \leq t_{q}, & q=2, \ldots, p-1, \\
\left\|\left[\begin{array}{c}
2 \\
s_{q}-M_{q}
\end{array}\right]\right\| \leq s_{q}+M_{q}, & q=2, \ldots, p-1, \\
w^{p-1} \cdot z_{o}^{p-1}+v^{p} \cdot x_{o}^{p} \leq t_{p}, & \\
\left\|\left[\begin{array}{l}
2 \\
s_{p}-M_{p}
\end{array}\right]\right\| \leq s_{p}+M_{p}, & \\
Z^{1} w^{1}+Y^{1} u^{1}+w_{0}^{1}-X^{1} v^{1} \leq 0, & \\
Z^{q} w^{q}+Y^{q} u^{q}+w_{0}^{q}-\left(Z^{q-1} w^{q-1}+X^{q} v^{q}\right) \leq 0, & q=2, \ldots, p-1, \\
Y^{p} u^{p}+w_{0}^{p}-\left(Z^{p-1} w^{p-1}+X^{p} v^{p}\right) \leq 0, & \\
M_{q} \geq 0, M_{q} \leq 1, & q=1, \ldots, p, \\
0 \leq v^{1} \cdot x_{o}^{1} \leq 1, & \\
0 \leq w^{q-1} \cdot z_{o}^{q-1}+v^{q} \cdot x_{o}^{q} \leq 1, & \\
0 \leq w^{p-1} \cdot z_{o}^{p-1}+v^{p} \cdot x_{o}^{p} \leq 1, & q=2, \ldots, p-1, \\
l_{q}^{o} \leq t_{q} \leq u_{q}^{o}, & q=1,2, \ldots, p, \\
L_{q}^{o} \leq s_{q} \leq U_{q}^{o}, & q=1, \ldots, p ; w_{0}^{q} \text { are free } \\
v^{q}, u^{q}, w^{q} \geq 0, &
\end{array}
$$

where

$$
\begin{aligned}
& M_{1}=w^{1} \cdot z_{o}^{1}+u^{1} \cdot y_{o}^{1}+w_{0}^{1}-E_{\min }^{1}\left(v^{1} \cdot x_{o}^{1}\right) \\
& M_{q}=w^{q} \cdot z_{o}^{q}+u^{q} \cdot y_{o}^{q}+w_{0}^{q}-E_{\min }^{q}\left(w^{q-1} \cdot z_{o}^{q-1}+v^{q} \cdot x_{o}^{q}\right), \quad q=2, \ldots, p-1, \\
& M_{p}=u^{p} \cdot y_{o}^{p}+w_{0}^{p}-E_{\min }^{p}\left(w^{p-1} \cdot z_{o}^{p-1}+v^{p} \cdot x_{o}^{p}\right)
\end{aligned}
$$

Proof. The proof is similar to the proof of Theorem 3.2.

Subsequently, the stages' efficiencies $E_{o}^{q},(q=1, \ldots, p)$ are obtained as follows:

$$
\begin{aligned}
E_{o}^{1} & =\frac{w^{1 *} \cdot z_{o}^{1}+u^{1 *} \cdot y_{o}^{1}+w_{0}^{1 *}}{v^{1 *} \cdot x_{o}^{1}}, \\
E_{o}^{q} & =\frac{w^{q *} \cdot z_{o}^{q}+u^{q *} \cdot y_{o}^{q}+w_{0}^{q *}}{w^{q-1 *} \cdot z_{o}^{q-1}+v^{q *} \cdot x_{o}^{q}}, \quad q=2, \ldots, p-1, \\
E_{o}^{p} & =\frac{u^{p *} \cdot y_{o}^{p}+w_{0}^{p *}}{w^{p-1 *} \cdot z_{o}^{p-1}+v^{p *} \cdot x_{o}^{p}},
\end{aligned}
$$

where $\left(v^{q *}, u^{q *}, w^{q *}, w_{0}^{q *}\right), q=1, \ldots, p$ are the optimal weights derived from model (4.2). Unlike the existing studies $[1,14]$ that could not maintain the relationship between the stages' efficiencies and the overall efficiencies in the multi-stage structure, obviously, here for $\mathrm{DMU}_{o}$ we have $E_{o}^{\mathrm{GNC}}=\prod_{q=1}^{p} E_{o}^{q}$. Notice that, this relationship also holds for models based on AED and MED in the cooperative context.

\section{NUMERICAL EXAMPLES}

In this section, we consider two numerical examples to illustrate the advantages of the proposed approaches. 
TABLE 2. Overall efficiency evaluation with different models.

\begin{tabular}{lllll}
\hline \hline & Model (2.1) & Efficiency of model (2.5) & Efficiency of model $(2.20)$ & Efficiency of model (3.2) \\
\cline { 2 - 5 } DMUs & $E^{\mathrm{BCC}}$ & $E^{\mathrm{AC}}$ & $E^{\mathrm{MC}}$ & $E^{\mathrm{NC}}$ \\
\hline 1 & 0.7594 & 0.5631 & 0.2260 & 0.2537 \\
2 & 1 & 0.9117 & 0.5150 & 0.6958 \\
3 & 1 & 0.8747 & 0.6167 & 0.6203 \\
4 & 1 & 0.5096 & 0.1099 & 0.1167 \\
5 & 1 & 0.6667 & 0.1332 & 0.1425 \\
6 & 1 & 0.9687 & 0.3640 & 0.8669 \\
7 & 1 & 0.9121 & 0.6319 & 0.6615 \\
8 & 1 & 0.9787 & 0.8901 & 0.9083 \\
9 & 1 & 0.8347 & 0.4285 & 0.5054 \\
10 & 1 & 0.8841 & 0.6438 & 0.6478 \\
11 & 1 & 1 & 0.8545 & 0.7612 \\
12 & 1 & 0.8743 & 0.6782 & 0.7028 \\
13 & 1 & 1 & 0.8092 & 0.8651 \\
14 & 1 & 0.8333 & 0.4326 & 0.5861 \\
15 & 1 & 0.6960 & 0.2553 & 0.2656 \\
16 & 1 & 0.6301 & 0.2885 & 0.2958 \\
17 & 1 & 0.8354 & 0.4929 & 0.4839 \\
18 & 1 & 0.9662 & 0.7730 & 0.5375 \\
19 & 1 & 0.8914 & 0.3682 & 0.4108 \\
20 & 1 & 0.8120 & 0.3829 & 0.3153 \\
21 & 1 & 0.7843 & 0.3152 & 1 \\
22 & 1 & 1 & 0.5764 & 0.8484 \\
23 & 1 & 0.9473 & 0.7997 & 0.8506 \\
24 & 1 & 0.9971 & 0.3104 & 0.6045 \\
25 & 1 & 0.8348 & 0.5939 & 0.3427 \\
26 & 1 & 0.6292 & 0.3495 & 0.3042 \\
27 & 0.8492 & 0.6887 & 0.2921 & 0.3285 \\
28 & 1 & 0.5542 & 0.2138 & 0.2739 \\
29 & 1 & 0.6351 & 0.3467 & \\
30 & 1 & 0.6887 & 0.2039 & \\
\hline
\end{tabular}

\section{- Example 1.}

The data for this example is taken from Despotis et al. [14] consists of 30 DMUs with a three-stage structure, as depicted in Figure 1. The breakdown points of each stage are obtained as $\left(E_{\min }^{1}, E_{\min }^{2}, E_{\min }^{3}\right)=$ $(0.2698,0.3853,0.4149)$ by $\mathrm{Du}$ et al. [16] method. Table 2 shows the overall efficiency of four different models. The second column indicates the efficiency scores of BCC model (2.1), where the internal structure is neglected. In the third and forth columns, we can see the overall efficiency derived from AED model (2.5) and MED model (2.20), respectively. The last column of Table 2 also shows the overall efficiency scores derived from model (3.2). The BCC model shows the efficiency scores of 28 DMUs equal to one, while by considering the internal performance of system in cooperative model (2.5), only three DMUs (11, 13 and 22 ) and in model (3.2), only DMU22 are efficient. Also, DMU8 is the most efficient DMU in cooperative model (2.20). Further, we can see in Table 2 that DMU1 has the lowest efficiency score in model (2.1), in contrast to the cooperative models (2.5) and (2.20) and bargaining model (3.2) indicate DMU4 as the most inefficient DMU. Figure 3 also shows the overall efficiency distribution of four models. 


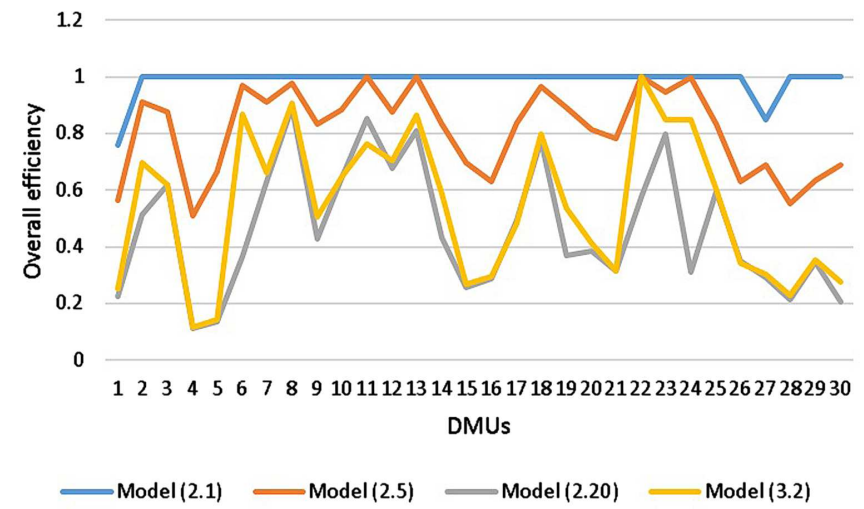

Figure 3. Change trend of the overall efficiency in different models.

The results of the stages' efficiencies are reported in Table 3. Columns $2-4$ and columns $5-7$ show the stages' efficiencies derived from the weights of AED and MED models in the coopeartive context, respectively. The results show that AED and MED scores are almost consistent.

Also, the stages' efficiency scores arising from the weights of bargaining model (3.2) are given in the last three columns of Table 3. Although the results of model (3.2) show a significant decrease in the number of efficient units, it has led to equilibrium between stages' efficiency. For instance, the number of efficient DMUs of stage 2 has been decreased from 14 DMUs in the cooperative model (2.5) to 7 units in the bargaining model (3.2). While we can see the efficiency scores of stage 3 are improved. Unlike both cooperative models, the bargaining model (3.2) makes an equilibrium between the stages. In general, depending on the managers' targets, whether to maximize efficiency or to achieve an equilibrium, each of these two approaches may be useful.

- Example 2.

The data set for this example is taken from Tone and Tsutsui [38] consists of 10 vertically integrated power companies in the US with three stages: Generation, Transmission and Distribution. The number of employees $\left(x^{1}\right)$ is stage 1's input to genetate electric power $\left(z^{1}\right)$, which is output from Generation stage and input to Transmission stage. Also, the number of employees $\left(x^{2}\right)$ as another input of stage 2 produces electric power sold to large customers $\left(y^{2}\right)$. In addition, electric power sent $\left(z^{2}\right)$ as output from the Transmission stage is inputted to stage 3 and alongside the number of employees $\left(x^{3}\right)$ generate electric power sold to small customers $\left(y^{3}\right)$. Note that this three-stage process is a special case of the one given in Figure 1 by removing $y^{1}$. The breakdown points of each stage are obtained as $\left(E_{\min }^{1}, E_{\min }^{2}, E_{\min }^{3}\right)=(0.1722,0.5432,0.2134)$ by Du et al. [16] method. Also, the overall efficiency of different models are summarized in Table 4. The second column of this table shows the results of classical BCC model where the internal structure and intermediate product are neglected. The overall efficiencies based on the network models (2.5), (2.20) and (3.2) are reported in columns 3-5 of Table 4, respectively. We see 8 DMUs (No. 1, 3, 4, 5, 7, 8, 9 and 10) in model (2.1) that are efficient, while the results of models (2.5), (2.20) and (3.2) show that none of the DMUs are efficient. Table 5 also reports the stages' efficiency scores of three different models in the presence of intermediate products. Columns 2-4 show the stages' efficiencies of the AED model (2.5). Also, we can see the stages' efficiency results derived from model (2.20) in columns $5-7$. The last three columns of this table also shows the efficiency scores of model (3.2). For example, for DMUs 2, 6, 7 and 9, we can see that $E^{2}$ of (3.2) is higher than $E^{2}$ of both cooperative models.

Moreover, we see that compared with two cooperative models (2.5) and (2.20), in spite of the efficiency decrease of some DMUs, the second stages' efficiencies mean are increased in the bargaining model (3.2). Indeed, using the bargaining game approach leads to increase transmission in the power companies. As the 
TABLE 3. Stages' efficiencies evaluation with different models.

\begin{tabular}{|c|c|c|c|c|c|c|c|c|c|}
\hline \multirow[b]{2}{*}{ DMUs } & \multicolumn{3}{|c|}{ Efficiency of model (2.5) } & \multicolumn{3}{|c|}{ Efficiency of model (2.20) } & \multicolumn{3}{|c|}{ Efficiency of model (3.2) } \\
\hline & $E^{1}$ & $E^{2}$ & $E^{3}$ & $E^{1}$ & $E^{2}$ & $E^{3}$ & $E^{1}$ & $E^{2}$ & $E^{3}$ \\
\hline 1 & 0.5384 & 0.6367 & 0.5141 & 0.6395 & 0.5469 & 0.6463 & 0.5101 & 0.6081 & 0.8177 \\
\hline 2 & 0.7381 & 1 & 1 & 0.5859 & 0.8943 & 0.9830 & 0.6958 & 1 & 1 \\
\hline 3 & 1 & 1 & 0.6242 & 1 & 1 & 0.6167 & 0.9999 & 1 & 0.6204 \\
\hline 4 & 0.3392 & 0.4596 & 0.7299 & 0.3094 & 0.5028 & 0.7063 & 0.3293 & 0.5029 & 0.7047 \\
\hline 5 & 0.0001 & 1 & 1 & 0.2691 & 0.4948 & 1 & 0.3000 & 0.4748 & 1 \\
\hline 6 & 1 & 1 & 0.9060 & 0.5940 & 0.9649 & 0.6350 & 0.9638 & 1 & 0.8995 \\
\hline 7 & 0.7364 & 1 & 1 & 0.6797 & 0.9297 & 1 & 0.6938 & 0.9535 & 1 \\
\hline 8 & 0.9361 & 1 & 1 & 0.9652 & 1 & 0.9318 & 0.9689 & 1 & 0.9375 \\
\hline 9 & 1 & 1 & 0.5041 & 0.8941 & 0.8476 & 0.5655 & 0.8888 & 1 & 0.5686 \\
\hline 10 & 1 & 1 & 0.6524 & 1 & 1 & 0.6438 & 1 & 1 & 0.6478 \\
\hline 11 & 1 & 1 & 1 & 1 & 0.9182 & 0.9306 & 0.9752 & 0.8881 & 0.8789 \\
\hline 12 & 0.8724 & 0.9496 & 0.8009 & 0.8186 & 0.8287 & 0.9997 & 0.8291 & 0.8477 & 1 \\
\hline 13 & 1 & 1 & 1 & 0.9921 & 0.8490 & 0.9607 & 0.9929 & 0.8916 & 0.9772 \\
\hline 14 & 1 & 0.8819 & 0.6181 & 0.9828 & 0.7303 & 0.6028 & 1 & 0.7850 & 0.7466 \\
\hline 15 & 0.6142 & 0.6519 & 0.8220 & 0.5026 & 0.6127 & 0.8292 & 0.5049 & 0.6306 & 0.8341 \\
\hline 16 & 0.5243 & 0.5551 & 0.8107 & 0.5814 & 0.6193 & 0.8013 & 0.5932 & 0.6231 & 0.8000 \\
\hline 17 & 0.7713 & 0.7839 & 0.9511 & 0.6971 & 0.7414 & 0.9537 & 0.6584 & 0.8493 & 0.8653 \\
\hline 18 & 1 & 0.8985 & 1 & 0.9999 & 0.7732 & 0.9999 & 1 & 0.7963 & 1 \\
\hline 19 & 0.6742 & 1 & 1 & 0.5347 & & 0.9603 & 0.6368 & 0.8441 & 1 \\
\hline 20 & 0.4361 & 1 & 1 & 0.3834 & 0.9988 & 0.9998 & 0.4108 & 1 & 1 \\
\hline 21 & 0.3528 & 1 & 1 & 0.3204 & 1 & 0.9837 & 0.3401 & 0.9875 & 0.9389 \\
\hline 22 & 1 & 1 & 1 & 0.7120 & 0.9711 & 0.8337 & 1 & 1 & 1 \\
\hline 23 & 0.9992 & 0.9962 & 0.8465 & 0.9970 & 0.9669 & 0.8295 & 0.9999 & 0.9996 & 0.8488 \\
\hline 24 & 0.9981 & 0.9961 & 0.9970 & 0.8786 & 0.6272 & 0.5633 & 0.9809 & 0.9538 & 0.9092 \\
\hline 25 & 1 & 0.7443 & 0.7600 & 1 & 0.7099 & 0.8366 & 1 & 0.7182 & 0.8417 \\
\hline 26 & 0.7264 & 0.7477 & 0.4136 & 0.7080 & 0.8367 & 0.5899 & 0.7206 & 0.7106 & 0.6693 \\
\hline 27 & 0.5720 & 0.7627 & 0.7313 & 0.5323 & 0.7445 & 0.7371 & 0.5451 & 0.7581 & 0.7361 \\
\hline 28 & 0.0807 & 0.5818 & 1 & 0.3454 & 0.6192 & 1 & 0.3715 & 0.6152 & 1 \\
\hline 29 & 0.1458 & 0.7596 & 1 & 0.4320 & 0.8024 & 1 & 0.4414 & 0.7988 & 1 \\
\hline 30 & 0.4581 & 0.6079 & 1 & 0.3886 & 0.5331 & 0.9842 & 0.4356 & 0.6287 & 1 \\
\hline
\end{tabular}

TABLE 4. Change trend of overall efficiency in different models.

\begin{tabular}{|c|c|c|c|c|}
\hline & Model (2.1) & Additive model (2.5) & Multiplicative model (2.20) & Bargaining model (3.2) \\
\hline DMUs & $E^{\mathrm{BCC}}$ & $E^{\mathrm{AC}}$ & $E^{\mathrm{MC}}$ & $E^{\mathrm{NC}}$ \\
\hline 1 & 1 & 0.7750 & 0.3278 & 0.4501 \\
\hline 2 & 0.6301 & 0.6353 & 0.0612 & 0.1318 \\
\hline 3 & 1 & 0.9997 & 0.7915 & 0.9457 \\
\hline 4 & 1 & 0.7656 & 0.2354 & 0.2525 \\
\hline 5 & 1 & 0.7180 & 0.1916 & 0.1922 \\
\hline 6 & 0.7693 & 0.8337 & 0.5227 & 0.6425 \\
\hline 7 & 1 & 0.8343 & 0.5584 & 0.5620 \\
\hline 8 & 1 & 0.8815 & 0.6656 & 0.6500 \\
\hline 9 & 1 & 0.8911 & 0.9075 & 0.9878 \\
\hline 10 & 1 & 0.6349 & 0.1706 & 0.1752 \\
\hline
\end{tabular}


TABLE 5. Change trend of stages' efficiencies in different models.

\begin{tabular}{llllllllll}
\hline \hline & \multicolumn{3}{c}{ Additive model $(2.5)$} & \multicolumn{3}{c}{ Multiplicative model (2.20) } & \multicolumn{3}{c}{ Bargaining model (3.2) } \\
\cline { 2 - 10 } DMUs & $E^{1}$ & $E^{2}$ & $E^{3}$ & $E^{1}$ & $E^{2}$ & $E^{3}$ & $E^{1}$ & $E^{2}$ & $E^{3}$ \\
\hline 1 & 0.6328 & 0.7590 & 0.9332 & 0.4878 & 0.6915 & 0.9719 & 0.6324 & 0.7326 & 0.9714 \\
2 & 0.2540 & 0.7771 & 0.8752 & 0.2596 & 0.3301 & 0.7145 & 0.2352 & 0.8883 & 0.6305 \\
3 & 1 & 1 & 0.9990 & 0.9999 & 0.9878 & 0.8014 & 0.9992 & 0.9986 & 0.9478 \\
4 & 0.2967 & 1 & 1 & 0.2555 & 0.9212 & 0.9999 & 0.2967 & 0.8512 & 0.9999 \\
5 & 0.2002 & 1 & 0.9537 & 0.2010 & 0.9993 & 0.9539 & 0.2014 & 0.9997 & 0.9544 \\
6 & 1 & 0.6865 & 0.8147 & 0.9914 & 0.5631 & 0.9365 & 0.9994 & 0.7082 & 0.9078 \\
7 & 0.7091 & 0.8599 & 0.9339 & 0.6257 & 0.9742 & 0.9160 & 0.6061 & 1 & 0.9272 \\
8 & 0.7847 & 0.8599 & 1 & 0.7280 & 0.9145 & 0.9999 & 0.7106 & 0.9151 & 0.9996 \\
9 & 1 & 0.6881 & 0.9850 & 0.9991 & 0.9177 & 0.9898 & 0.9994 & 0.9996 & 0.9888 \\
10 & 0.2616 & 0.7333 & 0.9099 & 0.2426 & 0.8531 & 0.8243 & 0.2488 & 0.8540 & 0.8246 \\
\hline
\end{tabular}

results show, the second stage efficiency mean in models (2.5), (2.20) and (3.2) are 0.8364, 0.8152 and 0.8947, respectively. Each stage in the cooperative approach tries to achieve the weights to maximise its efficiency, but cannot affect the weights of other stages. In other words, each stage's weights are independent of the weights of the other stages. While in model based on the bargaining approach, each stage's weights may depend on the weights of the other stages.

\section{Conclusions}

Conventional DEA models ignore the internal structure of DMUs and treat them as black box. In this paper, to identify the internal performance of system, we studied a three-stage network structure containing exogenous inputs and outputs. First, for the overall efficiency decomposition into stages' efficiency, we proposed cooperative AED and MED based models. Since the proposed models are nonlinear and the use of Charnes-Cooper transformation lead to parametric linear models, we used conic relaxation approach to solve them. On the other hand, in order to make an equilibrium based on the negotiation between the stages in the production process, we applied Nash bargaining game approach on the cooperative model that besides maintaining advantages of the cooperative model, provides a unique and fair decomposition of the overall efficiency. For the proposed nonlinear bargaining model a conic relaxation is presented instead of a parametric approach used in [1] for a special structure. The results of both multiplicative cooperative and bargaining models on two numerical examples show that the overall efficiency is equal to the product of the stages' efficiencies. Also, the overall efficiency of additive cooperative model is equal to arithmetic mean of stages' efficiencies. In general, depending on the managers' targets in an organization, whether to maximize the stages' efficiencies or to made equilibrium and fair allocation, each of these two approaches can be applied.

Acknowledgements. The authors would like to thank all reviewers for their useful comments and suggestions and University of Guilan for partially supporting this research.

\section{REFERENCES}

[1] M. Amirkhan, H. Didehkhani, K. Khalili-Damghani and A. Hafezalkotob, Measuring performance of a three-stage network structure using data envelopment analysis and Nash bargaining game: a supply chain application. Int. J. Inf. Technol. Decis. Making 17 (2018) 1429-1467.

[2] J. Aparicio, J.L. Ruiz and I. Sirvent, Closest target and minimum distance to the Pareto-efficient frontier in DEA. J. Prod. 28 (2007) 209-218.

[3] R.D. Banker, A. Charnes and W.W. Cooper, Some models for estimating technical and scale inefficiencies in data envelopment analysis. Manage. Sci. 30 (1984) 1078-1092. 
[4] S. Boyd and L. Vandenberghe, Convex Optimization. Cambridge University Press, Cambridge (2004).

[5] I. Castelli, R. Pesenti and W. Ukovich, A classification of DEA models when the internal structure of the decision making unites is considered. Ann. Oper. Res. 173 (2010) 207-235.

[6] S.L. Chao, Integrating multi-stage data envelopment analysis and a fuzzy analytical hierarchical process to evaluate the efficiency of major global liner shipping companies. Maritime Policy Manage. 44 (2017) 496-511.

[7] A. Charnes and W.W. Cooper, Programming with linear fractional functions. Naval Res. Logistics Q. 9 (1962) $181-185$.

[8] A. Charnes, W.W. Cooper and E. Rhodes, Measuring the efficiency of decision making units. Eur. J. Oper. Res. 2 (1978) 429-444.

[9] K. Chen and J. Zhu, Second order cone programming approach to two-stage network data envelopment analysis. Eur. J. Oper. Res. 262 (2017) 231-238.

[10] Y. Chen, W.D. Cook, N. Li and J. Zhu, Additive efficiency decomposition in two-stage DEA. Eur. J. Oper. Res. 196 (2009) 1170-1176.

[11] Y. Chen, J. Du, H.D. Sherman and J. Zhu, DEA model with shared resources and efficiency decomposition. Eur. J. Oper. Res. 207 (2010) 339-349.

[12] K. Chen, W.D. Cook and J. Zhu, A conic relaxation model for searching for the global optimum of network data envelopment analysis. Eur. J. Oper. Res. 280 (2020) 242-253.

[13] W.D. Cook, J. Zhu, G.B. Bi and F. Yang, Network DEA: additive efficiency decomposition. Eur. J. Oper. Res. 207 (2010) 1122-1129.

[14] D.K. Despotis, D. Sotiros and G. Koronakos, A network DEA approach for series multi-stage processes. Omega 61 (2016) 35-48.

[15] T.S.H. Driessen, Cooperative Games, Solutions and Applications. Kluwer Academic Publishers, Dordrecht (1988).

[16] J. Du, L. Liang, Y. Chen, W.D. Cook and J. Zhu, A bargaining game model for measuring performance of two-stage network structures. Eur. J. Oper. Res. 210 (2011) 390-397.

[17] R. Färe and S. Grosskopf, Productivity and intermediate products: a frontier approach. Econ. Lett. 50 (1996) 65-70.

[18] R. Färe and S. Grosskopf, Network DEA. Soc.-Econ. Planning Sci. 34 (2000) 35-49.

[19] C. Guo, F. Wei and Y. Chen, A note on second order cone programming approach to two-stage network data envelopment analysis. Eur. J. Oper. Res. 263 (2017) 733-735.

[20] M. Hu and M. Fukushima, Multi-leader-follower games: models, methods and applications. J. Oper. Res. Soc. Jpn. 58 (2014) $1-23$.

[21] C.R. Johnson, Positive definite matrices. Am. Math. Mon. 77 (1970) 259-264.

[22] C. Kao, Efficiency decomposition in network data envelopment analysis: a relational model. Eur. J. Oper. Res. 192 (2009) 949-962.

[23] C. Kao, Efficiency decomposition for general multi-stage systems in data envelopment analysis. Eur. J. Oper. Res. 232 (2014) 117-124.

[24] C. Kao, A classification of slacks-based efficiency measures in network data envelopment analysis with an analysis of the properties possessed. Eur. J. Oper. Res. 270 (2018) 1109-1121.

[25] C. Kao and S.N. Hwang, Efficiency decomposition in two-stage data envelopment analysis: an application to non-life insurance companies in Taiwan. Eur. J. Oper. Res. 185 (2008) 418-429.

[26] C.-Y. Lee and A. Johnson, Two-dimensional efficiency decomposition to measure the demand effect in productivity analysis. Eur. J. Oper. Res. 216 (2012) 584-593.

[27] Y. Li, Y. Chen, L. Liang and J. Xie, DEA models for extended two-stage network structures. Omega 40 (2012) $611-618$.

[28] H. Li, C. Chen, W.D. Cook, J. Zhang and J. Zhu, Two-stage network DEA: Who is the leader? Omega 74 (2018) 15-19.

[29] L. Liang, W.D. Cook and J. Zhu, DEA models for two-stage processes: game approach and efficiency decomposition. Nav. Res. Logistics 55 (2008) 643-653.

[30] L. Liang, Z.Q. Li, W.D. Cook and J. Zhu, Data envelopment analysis efficiency in two-stage networks with feedback. IIE Trans. 43 (2011) 309-322.

[31] R. Mahmoudi, A. Emrouznejad and M. Rasti-Barzoki, A bagaining game model for performance assessment in network DEAconsidering sub-networks: areal case study in banking. Neural Comput. App. 31 (2019) 6429-6447.

[32] M.Z. Mahmoudabadi and A. Emrouznejad, Comprehensive performance evaluation of banking branches: a three-stage slacksbased measure (SBM) data envelopment analysis. Int. Rev. Econ. Finance 64 (2019) 359-376.

[33] J.F. Nash, The bargaining problem. Econ. J. Econ. Soc. 18 (1950) 155-162.

[34] J.F. Nash, Two-person cooperative games, Econ. J. Econ. Soc. 21 (1953) 128-140.

[35] K. Ritzberger, Foundations of Non-Cooperative Game Theory. Oxford University Press (2002).

[36] M. Tavana and K. Khalili-Damghani, A new two-stage Stackelberg fuzzy data envelopment analysis model. Measurement 53 (2014) $277-296$.

[37] M. Tavana, M.A. Kaviani, D.D. Caprio and B. Rahpeyma, A two-stage data envelopment analysis model for measuring performance in three-level supply chains. Measurement $\mathbf{7 8}$ (2016) 322-333.

[38] K. Tone and M. Tsutsui, Network DEA: a slacks-based measure approach. Eur. J. Oper. Res. 197 (2009) $243-252$.

[39] J. Wu, L. Liang, F. Yang and H. Yan, Bargaining game model in the evaluation of decision making units. Expert Syst. App. 36 (2009) 3357-4362. 
[40] J. Wu, Q. Zhu, X. Ji, J. Chu and L. Liang, Two-stage network processes with shared resources and resources recovered from undesirable outputs. Eur. J. Oper. Res. 251 (2016) 182-197.

[41] H. Wu, K. Lv, L. Liang and H. Hu, Measuring performance of sustainable manufacturing with recyclablee waste: a case from China's iron and steel industry. Omega 66 (2017) 38-47.

[42] L. Zhang and K. Chen, Hierarchical network systems: an application to high-technology industry in China. Omega 82 (2017) $118-131$.

[43] L. Zhang, C. Guo and F. Wei, Multistage network data envelopment analysis: semidefinite programming approach. J. Oper. Res. Soc. 70 (2019) 1284-1295.

[44] X. Zhou, Z. Xu, J. Chai, L. Yao, S. Wang and B. Lev, Efficiency evaluation for banking systems under uncertainty: a multiperiod three-stageDEA model. Omega 85 (2018) 68-82.

[45] Z. Zhuo, L. Sun, W. Yang, W. Liu and C. Ma, A bargaining game model for efficiency decomposition in the centralized model of two-stage systems. Comput. Ind. Eng. 64 (2013) 103-108.

\section{Subscribe to Open (S20) A fair and sustainable open access model}

This journal is currently published in open access under a Subscribe-to-Open model (S2O). S2O is a transformative model that aims to move subscription journals to open access. Open access is the free, immediate, online availability of research articles combined with the rights to use these articles fully in the digital environment. We are thankful to our subscribers and sponsors for making it possible to publish this journal in open access, free of charge for authors.

\section{Please help to maintain this journal in open access!}

Check that your library subscribes to the journal, or make a personal donation to the S2O programme, by contacting subscribers@edpsciences.org

More information, including a list of sponsors and a financial transparency report, available at: https://www. edpsciences.org/en/maths-s2o-programme 